\title{
The intermediate scale MSSM,the Higgs mass and F-theory unification
}

\author{
L.E. Ibáñez, ${ }^{a, b}$ F. Marchesano, ${ }^{b}$ D. Regalado ${ }^{b}$ and I. Valenzuela ${ }^{a, b}$ \\ ${ }^{a}$ Departamento de Fúsica Teórica, Universidad Autónoma de Madrid, \\ Cantoblanco, 28049 Madrid, Spain \\ ${ }^{b}$ Instituto de Física Teórica UAM-CSIC, Universidad Autónoma de Madrid, \\ Cantoblanco, 28049 Madrid, Spain \\ E-mail: luis.ibannez@uam.es, fernando.marchesano@csic.es, \\ diego.regalado@csic.es, irene.valenzuela@uam.es
}

ABSTRACT: Even if SUSY is not present at the Electro-Weak scale, string theory suggests its presence at some scale $M_{S S}$ below the string scale $M_{s}$ to guarantee the absence of tachyons. We explore the possible value of $M_{S S}$ consistent with gauge coupling unification and known sources of SUSY breaking in string theory. Within F-theory SU(5) unification these two requirements fix $M_{S S} \simeq 5 \times 10^{10} \mathrm{GeV}$ at an intermediate scale and a unification scale $M_{c} \simeq 3 \times 10^{14} \mathrm{GeV}$. As a direct consequence one also predicts the vanishing of the quartic Higgs SM self-coupling at $M_{S S} \simeq 10^{11} \mathrm{GeV}$. This is tantalizingly consistent with recent LHC hints of a Higgs mass in the region $124-126 \mathrm{GeV}$. With such a low unification scale $M_{c} \simeq 3 \times 10^{14} \mathrm{GeV}$ one may worry about too fast proton decay via dimension 6 operators. However in the F-theory GUT context SU(5) is broken to the SM via hypercharge flux. We show that this hypercharge flux deforms the SM fermion wave functions leading to a suppression, avoiding in this way the strong experimental proton decay constraints. In these constructions there is generically an axion with a scale of size $f_{a} \simeq M_{c} /(4 \pi)^{2} \simeq 10^{12} \mathrm{GeV}$ which could solve the strong $\mathrm{CP}$ problem and provide for the observed dark matter. The price to pay for these attractive features is to assume that the hierarchy problem is solved due to anthropic selection in a string landscape.

Keywords: Compactification and String Models, F-Theory, Supersymmetry Breaking, GUT

ARXIV EPRINT: 1206.2655 


\section{Contents}

1 Introduction 1

$2 \mathrm{SU}(5)$ and F-theory unification $\quad 4$

$\begin{array}{lll}3 & \text { Gauge coupling unification and hypercharge flux } & 6\end{array}$

4 The quartic coupling and the Higgs mass 10

$\begin{array}{lll}5 & \text { Higgs mass fine-tuning } & 15\end{array}$

$\begin{array}{lll}6 & \text { Proton decay } & 17\end{array}$

7 Other consequences $\quad 22$

$\begin{array}{lll}7.1 \text { Axions } & 22\end{array}$

$\begin{array}{lll}7.2 & \text { Cosmology } & 24\end{array}$

$\begin{array}{lll}7.3 & \text { Neutrino masses } & 24\end{array}$

8 High Scale SUSY versus Split SUSY 25

9 Final conclusions and outlook $\quad 26$

A Non SUSY SM Type II orientifolds with open string fluxes 28

$\begin{array}{ll}\text { B RGE solutions } & 30\end{array}$

C Wave functions and proton decay $\quad 32$

\section{Introduction}

The LHC is already providing us with very important information on the physics underlying the Standard Model (SM) symmetry breaking process. A first piece of information are the constraints on the mass of the Higgs particle which is either heavier than $600 \mathrm{GeV}$ or else confined to a region in the area $120-127 \mathrm{GeV}$. In fact after the $7 \mathrm{TeV}$ run there are important hints suggesting a Higgs mass in the region $124-126 \mathrm{GeV}$ from both CMS and ATLAS $[1,2]$. A second piece of information is the absence of any trace of physics beyond the Standard Model (BSM). In particular there is at present no sign of squark and gluinos below $1 \mathrm{TeV}$, at least if they decay via the standard R-parity conserving channels in the Minimal Supersymmetric Standard Model (MSSM).

A Higgs mass around $125 \mathrm{GeV}$ is in principle good news for supersymmetry. Indeed such a value is consistent with the MSSM which predicts a mass $<130 \mathrm{GeV}$ for its lightest 
Higgs scalar. On the other hand getting such a Higgs mass within the MSSM typically requires a very massive SUSY spectrum with e.g. squarks at least in the $3-10 \mathrm{TeV}$ region [311]. This massive spectrum requires in turn a fine-tuning of the parameters at the per-mil level. If after the run at $14 \mathrm{TeV}$ the LHC sees no sign of supersymmetry or any other new physics BSM, the required fine-tuning will increase further and we will have to consider seriously the possibility that indeed the Electro-Weak (EW) scale is fine-tuned and selected on anthropic grounds [12-25].

If the EW scale is fine-tuned and low-energy SUSY does not play a role in the hierarchy issue, one may think of resurrecting good old non-SUSY unified theories like SU(5). We have to recall however the limitations of non-SUSY unification. Unification of gauge coupling constants, which works so well with the MSSM, fails in the non-SUSY case. Furthermore in minimal SU(5) models the unification scale is around $10^{14}-10^{15} \mathrm{GeV}$ and the proton decays too fast via dimension 6 operators. We also loose the existence of a natural candidate for dark matter to replace the neutralinos in the MSSM.

There is however another hint telling us that a non-SUSY desert up to a unified SU(5) scale and a fine-tuning of the Higgs mass is unlikely. Indeed if a SM Higgs mass is around $125 \mathrm{GeV}$ one knows what is the value of the Higgs quartic coupling $\lambda\left(M_{E W}\right)$ at the EW scale. One can then extrapolate its value up in energies using the Renormalization Group Equations (RGE). Due to the large top quark Yukawa coupling $\lambda$ decreases at higher energies and in fact seems to vanish at a scale around $10^{9}-10^{12} \mathrm{GeV}$, with the precise scale depending on the precise value of the top-quark mass $m_{t}$ and the strong coupling constant $\alpha_{3}$ [26-34]. If this is the case the theory becomes metastable before reaching the unification scale.

In any event, supersymmetric or not, one expects any unified theory to be combined with gravitation into an ultraviolet complete theory. At present the best candidate for such completion is provided by string theory. It is thus natural to try to address the unification issue within the context of string theory. In the last few years an interesting embedding of the SU(5) unification idea into F-theory has been the subject of much work (for reviews see [35-39]). These so called F-theory GUTs have some similarities with standard SU(5) field theory models but differ in some important aspects. Thus, e.g., the breaking of $\mathrm{SU}(5)$ down to the $\mathrm{SM}$ is produced by the presence of hypercharge fluxes in the compact dimensions instead of an explicit Higgs mechanism. As we will see this leads to several physical effects on both the gauge and Yukawa couplings which modify several aspects of field theory GUTs.

In the present paper we address the embedding of the SM or its SUSY version into the scheme of SU(5) F-theory unification without any prejudice about the size of the SUSY breaking scale $M_{S S}$. At this scale it is assumed that soft terms break the SUSY SM ${ }^{1}$ into the minimal SM. We study what the scale of unification $M_{c}$ and SUSY breaking $M_{S S}$ should be in order to obtain 1) correct gauge coupling unification, 2) sufficiently suppressed proton decay and 3) consistency with a SM Higgs in the $124-126 \mathrm{GeV}$ region.

\footnotetext{
${ }^{1}$ The SUSY spectrum could be that of the MSSM of some extension with e.g. extra Higgs doublets or triplets, see below. Whenever we write MSSM we also mean this kind of extensions, which will require minimal modifications.
} 
F-theory unification has specific hypercharge flux threshold corrections [40-42] to the inverse couplings $\alpha_{i}\left(M_{c}\right)^{-1}$ which are proportional to the inverse string constant $g_{s}^{-1}$. At strong coupling they are suppressed and correct gauge coupling unification is obtained with the MSSM spectrum and $M_{S S}=1 \mathrm{TeV}$ in the usual way. On the other hand, if one wants to remain at weak coupling, the threshold corrections are too large and spoil MSSM unification (unless extra effects from particles beyond the MSSM are included). However leaving the SUSY breaking scale free with $M_{S S} \gg 1 \mathrm{TeV}$ one finds that the threshold corrections have the required form and size to yield correct gauge coupling unification without the addition of any extra particle beyond the MSSM content. We argue that there are three independent arguments suggesting $M_{S S}$ is at an intermediate scale $M_{S S} \simeq$ $5 \times 10^{10} \mathrm{GeV}$ with gauge unification then fixing a unification scale $M_{c} \simeq 3 \times 10^{14} \mathrm{GeV}$. First, SUSY breaking induced by closed string fluxes gives rise generically to soft terms of order $M_{S S} \simeq M_{c}^{2} /\left(\alpha_{G}^{1 / 2} M_{p}\right) \simeq 5 \times 10^{10} \mathrm{GeV}$. Second, if indeed the SM Higgs self-coupling vanishes at a scale of order $10^{11} \mathrm{GeV}$, as seems to be implied by a $124-126 \mathrm{GeV}$ Higgs, this may be an indication of a SUSY boundary condition $\lambda\left(M_{S S}\right)=\frac{1}{8}\left(g_{1}^{2}+g_{2}^{2}\right) \cos ^{2} 2 \beta$ with $\tan \beta\left(M_{S S}\right) \simeq 1$. We show that this boundary condition is quite generic in string constructions in which a Higgs field $H_{S M}=\sin \beta H_{u}-\cos \beta H_{d}^{*}$ is fine-tuned to be massless. Finally, in F-theory SU(5) GUT's there is a natural candidate for a string axion with decay constant $f_{a} \simeq M_{c} /\left(16 \pi^{2}\right)$, which is in the right region $\simeq 10^{12} \mathrm{GeV}$ for axionic dark matter if $M_{c} \simeq 3 \times 10^{14} \mathrm{GeV}$.

In this $\mathrm{ISSB}^{2}$ framework the unification scale is relatively low, $M_{c} \simeq 3 \times 10^{14} \mathrm{GeV}$ and one may worry about fast proton decay via dimension 6 operators. Again, the fact that the breaking of the SU(5) symmetry down to the SM proceeds due to a hypercharge flux background rather than a Higgs mechanism modifies the expectations. Indeed, in field theory the gauge coupling of the $\mathrm{SU}(5) X, Y$ gauge bosons to fermions remains unchanged before and after symmetry breaking. However if symmetry breaking is induced by hypercharge fluxes, the $X, Y$ coupling to fermions may be substantially suppressed due to the fact that the profile of the corresponding wave functions is modified. We describe this novel effect in detail by using local F-theory wave-functions of SU(5) matter fields.

The outline of this paper is as follows. In the next section we present a brief review of F-theory SU(5) unification with emphasis on the particular aspects which play a role in the following sections. In section 3 we discuss gauge coupling unification and the scales of SUSY breaking $M_{S S}$ and unification $M_{c}$ naturally arising. In section 4 we discuss the value of the quartic Higgs coupling at $M_{S S}$ within the context of string compactifications, describing how $\tan \beta \simeq 1$ comes naturally. In the following section we discuss how the fine-tuning of a massless SM Higgs can proceed and the one-loop stability of the $\tan \beta \simeq 1$ boundary condition. In section 6 we address the issue of proton decay suppression and in section 7 we discuss other phenomenological consequences and in particular how an axion with an appropriate decay constant naturally appears within this framework. We briefly discuss the case of Split-SUSY in section 8 and leave section 9 for some final remarks and conclusions. Three appendices complement the main text.

\footnotetext{
${ }^{2}$ Intermediate Scale Supersymmetry Breaking.
} 


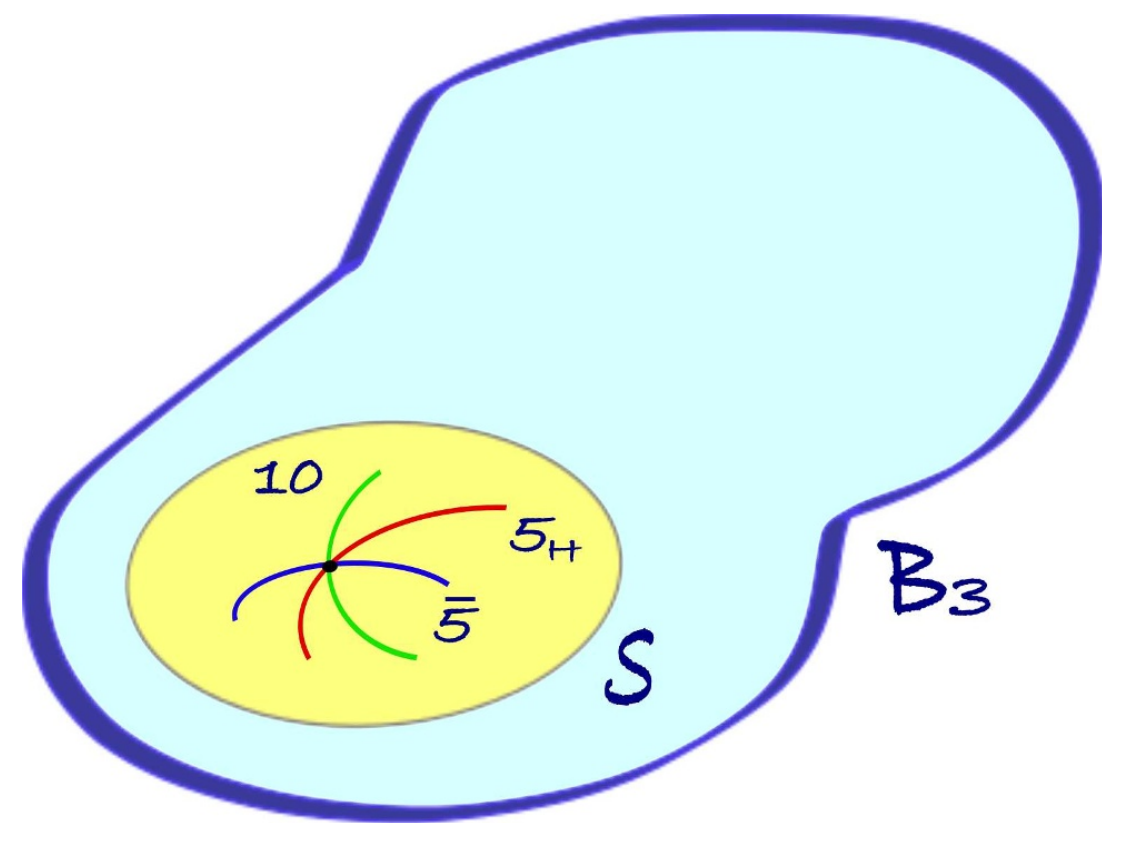

Figure 1. Scheme of an F-theory SU(5) GUT. The six extra dimensions are compactified on $B_{3}$ whereas the SU(5) degrees of freedom are localized on a 4-cycle submanifold $S$. The gauge bosons live on the bulk of $S$ but the chiral multiplets are localized on complex matter curves. At the intersection of two matter curves with a Higgs curve a Yukawa coupling develops.

\section{$2 \quad \mathrm{SU}(5)$ and F-theory unification}

F-theory [43-45] may be considered as a non-perturbative extension of Type IIB orientifold compactifications with 7-branes. This class of compactifications have two main phenomenological virtues compared to other string constructions [35-39]). First, in Type IIB compactifications it is well understood how moduli could be fixed in the presence of closed string fluxes and non-perturbative effects. Secondly, particularly within F-theory, GUT symmetries like SU(5) appear allowing for a correct structure of fermion masses (in particular a sizeable top quark mass). Here we just review a few concepts which are required for the understanding of the forthcoming sections. Our general discussion applies both to perturbative Type IIB and their F-theory extensions but we will refer to them as F-theory constructions for generality.

In Type IIB orientifold/F-theory unified models the SU(5) symmetry arises from the worldvolume fields of five 7-branes with their extra 4 dimensions wrapping a 4 -cycle $S$ inside a six dimensional compact manifold $B_{3}$, see figure 1 . The matter fields transforming in 10plets and 5-plets have their wave functions in extra dimensions localized on complex curves, the so called matter curves. These matter curves, which have two real dimensions, may be understood as intersections of the SU(5) 7-branes with extra U(1) 7-branes wrapping other 4-cycles in $B_{3}$. 
In order to get an idea of the relevant mass scales one can use results from perturbative Type IIB orientifolds. The string scale $M_{s}=\alpha^{\prime-1 / 2}$ is related to the Planck scale $M_{p}$ by [35]

$$
M_{p}^{2}=\frac{8 M_{s}^{8} V_{6}}{(2 \pi)^{6} g_{s}^{2}}
$$

where $V_{6}$ is the volume of the 6-manifold $B_{3}$ and $g_{s}$ in the string coupling constant. $\alpha^{\prime}$ is the inverse string tension. Note that one can lower $M_{s}$ by having a large volume $V_{6}$ (or decreasing $g_{s}$ ), so that the string scale is in principle a free parameter.

Now, the volume $V_{4}$ of the 4 -fold $S$ which is wrapped by the 7-branes is independent from the overall volume of $B_{3}$. This volume however is related to the inverse GUT coupling constant $\alpha_{G}$. In particular one has at tree level

$$
\frac{1}{\alpha_{G}}=4 \pi R e f_{\mathrm{SU}(5)}=\frac{1}{8 \pi^{4} g_{s}}\left(\frac{V_{4}}{\alpha^{\prime 2}}\right)
$$

with $f_{\mathrm{SU}(5)}$ the gauge kinetic function. Parametrizing $V_{4}=\left(2 \pi R_{c}\right)^{4}$ one then has

$$
M_{c}=M_{s}\left(\frac{\alpha_{G}}{2 g_{s}}\right)^{1 / 4}
$$

where we define $M_{c}=1 / R_{c}$. This is slightly below the string scale (i.e. for $g_{s}=1 / 2$ and $\alpha_{G}=1 / 38$ one has $\left.M_{c} \simeq 0.4 M_{s}\right)$. This scale $M_{c}$ can be identified with the GUT scale at which $\mathrm{SU}(5)$ is broken down to the SM. Indeed in F-theory GUTs there are no adjoint Higgs multiplets nor discrete Wilson lines available and it is a hypercharge flux background $<F_{Y}>\neq 0$ which does the job [46-49]. These fluxes go through holomorphic curves $\Sigma$ inside $S$ and they are quantized, $\int_{\Sigma} F_{Y}=$ integer. Thus on dimensional grounds one has $<F_{Y}>\simeq 1 / R_{c}^{2}=M_{c}^{2}$ and indeed one can identify $M_{c}$ with the GUT scale. ${ }^{3}$

We want to consider here the case in which slightly below the unification scale we have unbroken $N=1$ SUSY with an MSSM spectrum (or some slight generalization, see below). One reason for that assumption is that such class of vacua have no tachyons which could cause any premature instability in the theory. We will find additional a posteriori justifications for such an option in the forthcoming sections. We will however allow for SUSY to be broken in the MSSM sector at a scale $M_{S S}$ to be determined. It is however important to realize that there is a natural scale of SUSY breaking in Type IIB/F-theory compactifications.

Indeed, a most natural source of SUSY breaking is the presence of closed string fluxes in such vacua. More precisely, it is well known that e.g. generic RR and NS 3-form fluxes $G_{3}$ in Type IIB orientifolds induce SUSY-breaking soft terms [50-56]. These are also the fluxes which play a prominent role in the fixing of the moduli in these vacua. Since these

\footnotetext{
${ }^{3}$ Hypercharge fluxes have an additional use in F-theory GUT's. Indeed, by appropriately choosing these open string fluxes one can get doublet-triplet splitting in the SU(5) Higgs 5-plet, see refs. [35-39, 46-49] for details. However, as we will remark later, doublet-triplet splitting is not strictly necessary in our scheme, and so the constraints that are usually required on the hypercharge flux in order to achieve doublet-triplet splitting can be relaxed in our setup.
} 
fluxes live in the full $\mathrm{CY}$ and are quantized on 3-cycles, the said soft terms scale like $G_{3} \simeq c \alpha^{\prime} /\left(V_{6}^{1 / 2}\right)$. One thus finds for the size of soft terms [35]

$$
M_{S S} \simeq \frac{g_{s}^{1 / 2}}{\sqrt{2}} G_{3}=\frac{c g_{s}^{1 / 2}}{\sqrt{2}} \frac{\alpha^{\prime}}{V_{6}^{1 / 2}}=\frac{c M_{s}^{2}}{M_{p}}
$$

with c some fudge factor. Taking $c=1$ and taking into account eq. (2.3) one thus has an estimation for $M_{S S}{ }^{4}$

$$
M_{S S}=\frac{\left(2 g_{s}\right)^{1 / 2}}{\alpha_{G}^{1 / 2}} \frac{M_{c}^{2}}{M_{p}} .
$$

We will discuss other possible sources of SUSY breaking in section 4.

Although it will not play a relevant role in our discussion, let us briefly mention how the Yukawa couplings appear in the framework of F-theory unification. As we said the matter multiplets of the MSSM are confined in complex matter curves within the 4-fold $S$. Yukawa couplings appear at triple intersection points in $S$ in which two matter curves involving 10-plets and 5-plets cross with a matter curve containing the Higgs 5-plets, see figure 1. The Yukawa couplings may be computed as in standard Kaluza-Klein compactifications from triple overlap integrals of the form

$$
\mathcal{Y}_{D, L}^{i j}=\int_{S} \Psi_{10}^{i} \Psi_{\overline{5}}^{j} \Phi_{H_{D}} \quad \mathcal{Y}_{U}^{i j}=\int_{S} \Psi_{10}^{i} \Psi_{10}^{j} \Phi_{H_{U}} .
$$

where $i, j$ are family indices. The wave functions have a Gaussian profile so that one only needs local information about these wave functions around the intersection points in order to compute the Yukawa couplings. This local information may be extracted from the local equations of motion which may be solved so that quite explicit expressions for these wave functions may be obtained. We will use these local wave functions when discussing the proton decay suppression in section 6 .

As a summary we see that F-theory SU(5) unification allows for a general structure of scales $M_{S S}<M_{c}<M_{s}<M_{p}$. In what follows we will discuss how constraints from gauge coupling unification and the Higgs mass fix these scales.

\section{Gauge coupling unification and hypercharge flux}

In order to check for gauge coupling unification we will assume that at some scale $M_{c}$ the $\mathrm{SU}(5)$ symmetry is broken by hypercharge fluxes down to the SM group. We will asume that after this breaking the particle content is that of the MSSM (although we will allow for some variation below). However, unlike in the usual low energy SUSY scenario, we allow

\footnotetext{
${ }^{4}$ Note that setting $M_{s} \simeq 10^{11} \mathrm{GeV}$ one would obtain a scheme with soft terms around $1 \mathrm{TeV}$, which would be consistent with a SUSY solution of the hierarchy problem. However that would require also a string scale of order $M_{s} \simeq 10^{11} \mathrm{GeV}$ and MSSM gauge coupling unification would be lost. Alternatively one can set $M_{c} \simeq M_{s} \simeq 10^{16} \mathrm{GeV}$ consistent with MSSM gauge coupling unification if the effect of fluxes is somehow suppressed. Possible ways to suppress it would be assuming some fine-tuning in the flux or some warp factor leading to a flux dilution. This is the implicit assumption in models with flux induced SUSY breaking, $M_{s} \simeq 10^{16} \mathrm{GeV}$ and a standard SUSY solution to the hierarchy problem.
} 
the scale of SUSY breaking on the MSSM $M_{S S}$ to be a free parameter. We know that the standard MSSM prediction for gauge coupling unification [57-59] with $M_{S S} \simeq 1 \mathrm{TeV}$ is quite successful. On the other hand for $M_{S S} \simeq M_{c}$ we have the SM below $M_{c}$ and we know that coupling unification fails. On the basis of this one could conclude that gauge coupling unification forces $M_{S S}$ to be close to the weak scale. Interestingly enough, the breaking of the SU(5) symmetry via fluxes has a novel type of threshold corrections [40-42] compared to the field theory case, as we now describe. ${ }^{5}$ To leading order the gauge kinetic function for the $\mathrm{SU}(5)$ group within the 7-branes is given by the local Kähler modulus $T$ whose real part is proportional to $V_{4}$, consistently with eq. (2.2). However in the presence of hypercharge fluxes $F_{Y}$ the gauge kinetic functions get corrections [41]

$$
\begin{aligned}
4 \pi f_{\mathrm{SU}(3)} & =T-\frac{1}{2} \tau \int_{S} F_{a} \wedge F_{a} \\
4 \pi f_{\mathrm{SU}(2)} & =T-\frac{1}{2} \tau \int_{S}\left(F_{a} \wedge F_{a}+F_{Y} \wedge F_{Y}\right) \\
\frac{3}{5} 4 \pi f_{\mathrm{U}(1)} & =T-\frac{1}{2} \tau \int_{S}\left(F_{a} \wedge F_{a}+\frac{3}{5}\left(F_{Y} \wedge F_{Y}\right)\right) .
\end{aligned}
$$

where $\tau=\frac{1}{g_{s}}+i C_{0}$ is the complex dilaton and $F_{a}$ are fluxes along the $\mathrm{U}(1)$ contained in the $\mathrm{U}(5)$ gauge group of the 7 -branes which are needed for technical reasons but are not relevant in our discussion. It turns out that in order to get rid of exotic matter massless fields beyond those of the MSSM the topological condition $\int F_{Y} \wedge F_{Y}=-2$ should be fulfilled [46-49]. This implies that at the compactification scale one has the condition

$$
\frac{1}{\alpha_{1}\left(M_{c}\right)}=\frac{1}{\alpha_{2}\left(M_{c}\right)}+\frac{2}{3 \alpha_{3}\left(M_{c}\right)} .
$$

which is a generalization of the standard relationship $5 / 3 \alpha_{1}=\alpha_{2}=\alpha_{3}$. In addition one also obtains

$$
\frac{1}{g_{s}}=\frac{3}{5 \alpha_{1}\left(M_{c}\right)}-\frac{1}{\alpha_{3}\left(M_{c}\right)}=\frac{3}{5}\left(\frac{1}{\alpha_{2}\left(M_{c}\right)}-\frac{1}{\alpha_{3}\left(M_{c}\right)}\right) .
$$

Thus the size of the threshold corrections is determined by the inverse of the string coupling $g_{s}$. The corrections by themselves would imply an ordering of the size of the fine structure constants at $M_{c}$ given by

$$
\frac{1}{\alpha_{3}\left(M_{c}\right)}<\frac{1}{\alpha_{1}\left(M_{c}\right)}<\frac{1}{\alpha_{2}\left(M_{c}\right)} .
$$

We will be neglecting in what follows other possible sources of threshold corrections like the loop contributions of KK massive modes. The corrections in eq. (3.2) may in fact spoil the standard joining of gauge coupling constants in the MSSM, which works quite well, unless they are suppressed by assuming $g_{s} \gg 1$. Furthermore the above ordering of couplings goes in the wrong direction if one wanted to use such corrections to further improve the agreement with experiment [41].

\footnotetext{
${ }^{5}$ These corrections result from the expansion in powers of fluxes $F$ of the Dirac-Born-Infeld plus ChernSimmons (DBI+CS) action of the 7-branes, see e.g. [35] for a review.
} 
Interestingly enough, in our setting with undetermined $M_{S S}$ the corrections have just the required form and size to get consistency with gauge coupling unification without the addition of any extra matter field beyond the MSSM (see also ref. [60]). The one-loop renormalization group equations lead to the standard formulae

$$
\frac{1}{\alpha_{i}\left(M_{c}\right)}=\frac{1}{\alpha_{i}\left(M_{E W}\right)}-\frac{b_{i}^{N S}}{2 \pi} \log \frac{M_{S S}}{M_{E W}}-\frac{b_{i}^{S S}}{2 \pi} \log \frac{M_{c}}{M_{S S}}
$$

where $b_{i}^{N S}, b_{i}^{S S}$ are the one-loop beta-function coefficients of the SM and the MSSM respectively. These are given by $\left(b_{1}, b_{2}, b_{3}\right)^{N S}=(41 / 6,-19 / 6,-7)$ and $\left(b_{1}, b_{2}, b_{3}\right)^{S S}=(11,1,-3)$. Combining these equations and including the boundary condition (3.2) (which amounts to allowing for the above threshold corrections) one obtains

$$
\begin{aligned}
2 \pi\left(\frac{1}{\alpha_{1}\left(M_{E W}\right)}-\frac{1}{\alpha_{2}\left(M_{E W}\right)}-\frac{2}{3 \alpha_{3}\left(M_{E W}\right)}\right)= & \\
\quad=\left(b_{1}^{N S}-b_{2}^{N S}-\frac{2}{3} b_{3}^{N S}\right) \log \left(\frac{M_{S S}}{M_{E W}}\right) & +\left(b_{1}^{S S}-b_{2}^{S S}-\frac{2}{3} b_{3}^{S S}\right) \log \left(\frac{M_{c}}{M_{S S}}\right)
\end{aligned}
$$

In our case this yields

$$
\frac{44}{3} \log \frac{M_{S S}}{M_{E W}}+12 \log \frac{M_{c}}{M_{S S}}=2 \pi\left(\frac{1}{\alpha_{1}\left(M_{E W}\right)}-\frac{1}{\alpha_{2}\left(M_{E W}\right)}-\frac{2}{3 \alpha_{3}\left(M_{E W}\right)}\right) .
$$

This is displayed by the black line in figure 2 . We have used the central values of the couplings

$$
\begin{aligned}
\alpha_{3}\left(M_{E W}\right) & =0.1184 \pm 0.0007 \\
\alpha_{e m}^{-1}\left(M_{E W}\right) & =128.91 \pm 0.02 \\
\sin ^{2} \theta_{W}\left(M_{E W}\right) & =0.23120 \pm 0.00015 .
\end{aligned}
$$

One observes that one can get consistent unification for values of $M_{S S}$ up to slightly below $10^{14} \mathrm{GeV}$, which is required by the condition $M_{S S}<M_{c}$. The unification scale has also a lower bound at the same scale. If however we impose that SUSY breaking is induced by closed string fluxes with $M_{S S}=\left(\left(2 g_{s}\right)^{1 / 2} / \alpha_{G}^{1 / 2}\right) \frac{M_{c}^{2}}{M_{p}}$ as explained in the previous section, the values of both $M_{S S}$ and $M_{c}$ are fixed yielding

$$
M_{S S}=5 \times 10^{10} \mathrm{GeV} ; \quad M_{c}=3 \times 10^{14} \mathrm{GeV} .
$$

Thus one gets correct coupling unification consistent with closed string flux SUSY breaking for SUSY broken at intermediate scale $\simeq 10^{11} \mathrm{GeV}$ and a slightly low $\mathrm{SU}(5)$ unification scale of order $10^{14} \mathrm{GeV}$. This immediately poses an apparent problem with proton decay that we will deal with in section 6 .

It is also interesting to display the value of $g_{s}$ as a function of $M_{S S}$ from eq. (3.3). This is shown in figure 3. For the values in eq. (3.11) one finds $g_{s}=0.47$. This shows that the string coupling here is in a perturbative regime. ${ }^{6}$ On the other hand for values

\footnotetext{
${ }^{6}$ This is interesting information on the possible size of string instanton effects since they are typically of order $\exp \left(-2 \pi / g_{s}\right)$. This yields corrections of order $10^{-5}$ for $g_{s}=1 / 2$, which may be relevant to the generation of $\mu$-terms or neutrino masses, see below.
} 


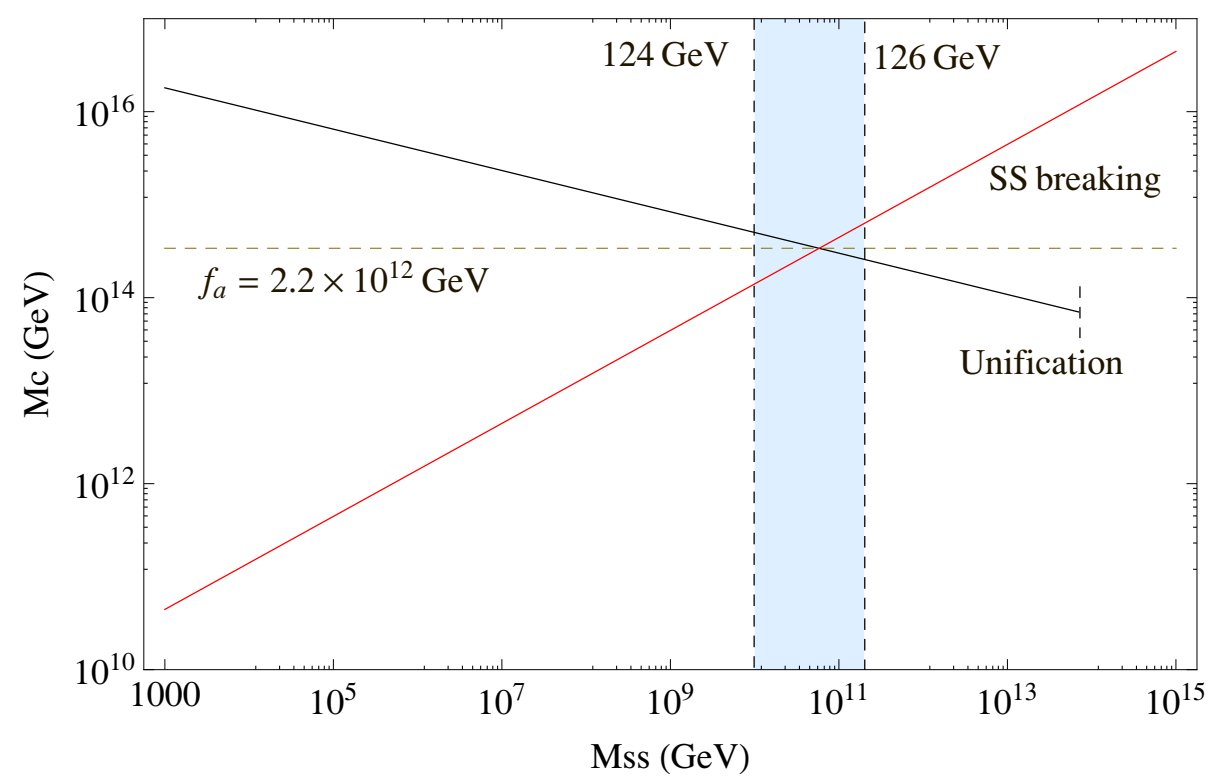

Figure 2. Constraints on $M_{S S}$ and $M_{c}$ from gauge coupling unification (black line) and closed string flux induced SUSY breaking (red line). The vertical (blue) band shows the region of $M_{S S}$ at which the SM Higgs coupling $\lambda$ vanishes for a Higgs mass in the range $124-126 \mathrm{GeV}$ and $m_{t}=173.2 \mathrm{GeV}$, as extracted from refs. [27, 29]. The horizontal line shows the value of the axion decay constant for the selected unification mass $M_{c}$.

$M_{S S} \simeq 1 \mathrm{TeV}$, corresponding to standard MSSM low-energy supersymmetry one needs $g_{s} \gg 1$. Note that in the context of F-theory the dilaton value $g_{s}$ varies over different locations in extra dimensions and may be large or small, so both situations are possible in F-theory GUTs.

Another interesting point is that the unification line in figure 2 does not change if in the region $M_{S S}-M_{c}$ there are incomplete SU(5) 5-plets, as equation (3.7) does not change. Thus for example the curve remains the same if the SU(5) partner of the Higgs doublets, the triplets $D, \bar{D}$ transforming like $(3,1,1 / 3)+$ c.c., remain in the spectrum below $M_{c}$. These triplets are potentially dangerous since their exchange give rise to dimension 6 proton decay operators. The rate is above experimental limits unless $M_{D} \geq 10^{11} \mathrm{GeV}$ [61], see section 6. That is why in GUTs one needs to perform some form of doublet-triplet splitting so that the Higgs fields remain light but the triplets are superheavy. In our case however these triplets will get a mass of order $M_{S S} \approx 10^{11} \mathrm{GeV}$ anyhow so they may be tolerated below $M_{c}$ and no doublet-triplet splitting is necessary. The presence of these triplets does however affect the size of the threshold corrections and $g_{s}$. In this case one gets typically smaller $g_{s}$ which slowly grows as $M_{S S}$ increases, see figure 3 . For $M_{S S} \simeq 10^{11} \mathrm{GeV}$ one gets $g_{s}=0.34$. 

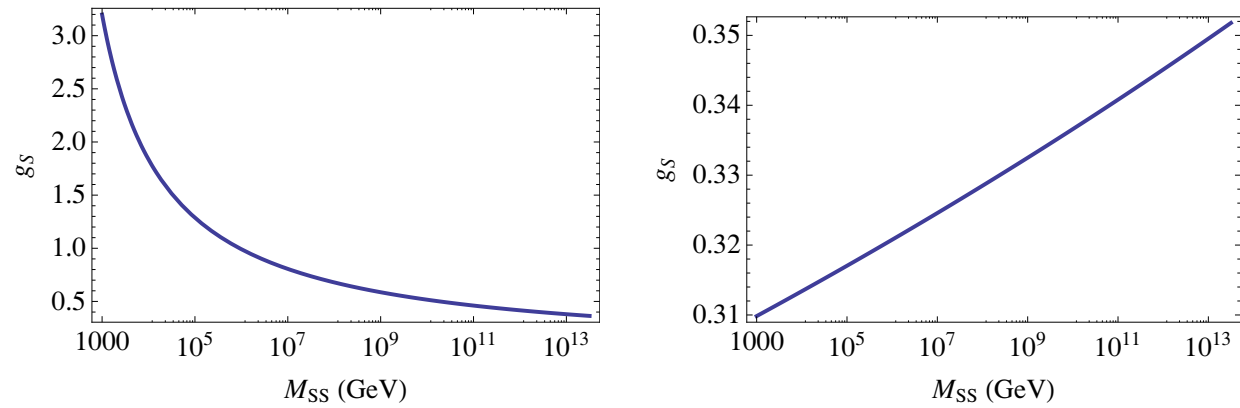

Figure 3. The string dilaton coupling constant versus $M_{S S}$ for consistent gauge coupling unification. Left: With an MSSM content in the region $M_{S S}-M_{c}$; Right: With an additional vector-like triplet set $D+\bar{D}$ in that region.

\section{The quartic coupling and the Higgs mass}

We have seen in the previous section how this ISSB framework is consistent both with gauge coupling unification and flux-induced SUSY breaking. Interestingly enough it has been recently found that if a non-SUSY SM Higgs is around $125 \mathrm{GeV}$, the SM RGE of the quartic self-coupling seems to drive it to a vanishing value at around $10^{11} \mathrm{GeV}$ or so (see e.g. $[27,28])$.

The question is whether there is any SUSY/string based scheme in which that happens naturally. Note that the Intermediate Scale SUSY Breaking (ISSB) described above corresponds to a variant of the High Scale SUSY Breaking (HSSB) scheme of Hall and Nomura in ref. [20]. This is just assuming a MSSM structure above a very large SUSY scale $M_{S S}$. All SUSY partners are heavy but there is still some imprint left of the High Scale SUSY in the Higgs sector. Indeed out of the two scalars $H_{u}, H_{d}$ in the MSSM only one linear combination remains light below $M_{S S}$, i.e.

$$
H_{S M}=\sin \beta H_{u}-\cos \beta H_{d}^{*}
$$

Then there is a quartic self-coupling $\lambda_{S S}\left|H_{S M}\right|^{4}$ with [18-20]

$$
\lambda_{S S}=\frac{1}{8}\left(g_{2}^{2}+\frac{3}{5} g_{1}^{2}\right) \cos ^{2} 2 \beta
$$

which is inherited from the D-term scalar potential of the MSSM. As we said it has been shown [27-34] that, starting at low-energies with a SM Higgs with a mass around 124$126 \mathrm{GeV}$ and running up the SM self-coupling $\lambda$ up in energies this coupling tends to zero around a scale $10^{9}-10^{11} \mathrm{GeV}$ (see figure 4 ). This would be consistent with the above High Scale SUSY Breaking scheme if at the scale $M_{S S}$ one had $\tan \beta= \pm 1$, so that $\lambda_{S S}\left(M_{S S}\right) \simeq 0$. 


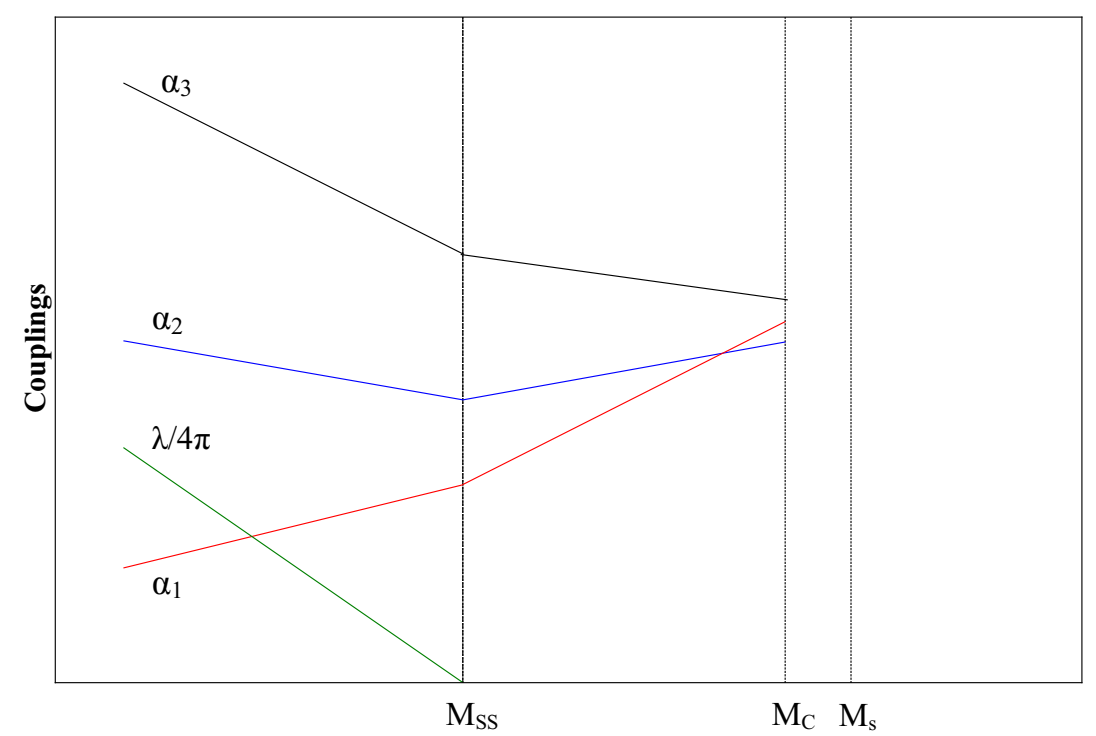

Figure 4. Structure of scales and the running of the gauge and Higgs coupling constants in this scheme.

An interesting question is thus under what conditions one naturally gets $\tan \beta \simeq \pm 1$. The general form of Higgs masses in the MSSM is ${ }^{7}$

$$
\left(H_{u}, H_{d}^{*}\right)\left(\begin{array}{cc}
m_{H_{u}}^{2} & m_{3}^{2} \\
m_{3}^{2} & m_{H_{d}}^{2}
\end{array}\right)\left(\begin{array}{c}
H_{u}^{*} \\
H_{d}
\end{array}\right) .
$$

where we will take $m_{3}^{2}$ real. The condition for a massless eigenvalue is $m_{3}^{4}=m_{H_{u}}^{2} m_{H_{d}}^{2}$. The massless eigenvector is then

$$
H_{S M}=\sin \beta H_{u} \mp \cos \beta H_{d}^{*}
$$

with $\sin \beta= \pm\left|m_{H_{d}}\right| / \sqrt{m_{H_{u}}^{2}+m_{H_{d}}^{2}}$. So in order to have a massless Higgs with $\tan \beta \simeq \pm 1$ one needs to have the conditions

$$
\begin{aligned}
m_{H_{u}}^{2} & =m_{H_{d}}^{2} \\
m_{3}^{4} & =m_{H_{u}}^{2} m_{H_{d}}^{2}
\end{aligned}
$$

We will take the negative sign in (4.4) from now on. The first condition points to an underlying symmetry under the exchange of $H_{u}$ and $H_{d}$, possibly slightly broken. The second condition does not necessarily imply any underlying symmetry, it is rather a finetuning constraint which has to be there anyhow if we want to get a light Higgs. So this could be selected on anthropic grounds.

\footnotetext{
${ }^{7}$ If in addition to the Higgs doublets there remain Higgs triplets $D, \bar{D}$ below $M_{c}$, similar mass matrices will appear for them. However there will be no anthropic selection of light scalar triplets. So doublet-triplet splitting would be purely anthropic.
} 
One can consider as a first option the direct construction of non-SUSY compactifications. Examples of this could be e.g. theories obtained from branes sitting at non-SUSY $\mathbf{Z}_{N}$ orbifold singularities (see e.g., ref. [62]). In this class of theories the particles in the spectrum, typically involving both fermions and scalars, have no SUSY partners to start with. These are however problematic since the spectrum generically contains tachyons from the closed string sector which destabilize the theory. So we will restrict ourselves to theories in which there is an underlying $N=1$ SUSY which is spontaneously broken to $N=0$. This will guarantee the absence of closed or open string tachyons from the start.

There are several possible sources for spontaneous SUSY breaking in the IIB/F-theory context which may arise from open or closed string fluxes ${ }^{8}$ which we discuss in turn.

\section{i) SUSY breaking terms and open string fluxes}

In Type IIB/F-theory in the large volume limit the Higgs fields will appear as KK zero modes. Open string fluxes, like the $F_{a}, F_{Y}$ mentioned above are in general present in order to generate chirality and symmetry breaking. These fluxes may induce also Higgs masses and SUSY-breaking terms as in eq. (4.3). We now review how such mass terms may appear in Type II toroidal orientifolds as discussed in [63]. In this reference a large class of non-SUSY Type IIA orientifolds with SM group and three chiral generations is discussed in terms of D6-branes intersecting at angles. These models may be converted into Type IIB orientifolds with D7-branes by the duality that relates intersection angles $\theta_{a b}$ between two branes $a, b$ into magnetic fluxes at their overlap, through the map $\theta_{a b}=\tan ^{-1}\left(2 \pi \alpha^{\prime} F_{a}\right)-\tan ^{-1}\left(2 \pi \alpha^{\prime} F_{b}\right)$. In these nonSUSY models (see appendix A for a short review) the Higgs fields appear from the exchange of open strings between an $\mathrm{SU}(2)$ stack of branes $(b)$ and a brane $(c)$ or its orientifold mirror $\left(c^{*}\right)$. The underlying torus is factorized as $\mathbf{T}^{2} \times \mathbf{T}^{2} \times \mathbf{T}^{2}$ and the branes $(b)$ and $(c),\left(c^{*}\right)$ are separated in the second torus by a distance $Z_{2}^{(b c)}\left(Z_{2}^{(b c)^{*}}\right)$. This means that the Higgs fields have a mass term proportional to this distance. In addition the magnetic fluxes induce non-SUSY mass contributions. One gets a structure (in the dilute flux limit) [63]

$$
\begin{aligned}
m_{H_{u}}^{2} & =m_{H_{d}}^{2}=\frac{Z_{2}^{(b c)}}{4 \pi^{2} \alpha^{\prime}} \\
m_{3}^{2} & =\left|\left(F_{b}-F_{c}\right)^{1}-\left(F_{b}-F_{c}\right)^{3}\right|
\end{aligned}
$$

where $F_{b}^{i}, F_{c}^{i}$ are fluxes from U(1)'s in the $b, c$ branes going through the first and third torus, see appendix A. Note that the first contributions correspond to a $\mu$-term and that it automatically implies eq. (4.5). This happens because one may understand $H_{u}$ and $H_{d}$ as coming from the same $N=2$ hypermultiplet before SUSY is broken by the fluxes. On the other hand the size of $m_{3}^{2}$ depends on the size and alignment of fluxes. For $\left(F_{b}-F_{c}\right)^{1}=\left(F_{b}-F_{c}\right)^{3}$ one recovers unbroken SUSY in the Higgs subsystem. The $\mathrm{SU}(2) \times \mathrm{U}(1)_{Y}$ D-term quartic selfcoupling is given by $\lambda=\left(g_{1}^{2}+g_{2}^{2}\right) / 8$ and, since this is a dimension 4 operator, it remains the same for $\left(F_{b}-F_{c}\right)^{1} \neq\left(F_{b}-F_{c}\right)^{3}$. In

\footnotetext{
${ }^{8}$ In the F-theory context both closed and open string fluxes have a common origin as $G_{4}$ fluxes.
} 
principle for fixed $Z_{2}^{b c}$ one can fine-tune the fluxes so that eq. (4.6) is met. Thus fine tuning of fluxes (or distance $Z_{2}^{(b c)}$ ) yields a massless Higgs multiplet corresponding to $\tan \beta=1$.

Note that the open string flux misalignment corresponds to a D-term SUSY breaking. This means that by itself this can only give SUSY breaking scalar masses but no gaugino masses (Higgssinos get SUSY masses for $Z_{2}^{b c} \neq 0$ ). Thus this class of SUSY breaking should be supplemented by further sources if below $M_{S S}$ we want to get just the content of the SM.

The above structure is generic and appears in any type II configuration where one can construct D-brane sectors with an $N=2$ hypermultiplet or a similar spectrum. In type IIB models with intersecting D7-branes or in F-theory GUTs such sectors arise quite naturally, since at the six-dimensional intersection of two 7-branes in flat space lives a $6 \mathrm{~d} N=1$ hypermultiplet that is equivalent to the $4 \mathrm{~d} N=2$ hypermultiplet of the construction above [64]. Hence, in order to reproduce the above structure for the Higgs sector, one may consider the case where the Higgs matter curve $\Sigma_{H}$ yields a non-chiral, $\mathrm{N}=2$ subsector of the theory. As the presence of a net flux over a matter curve induces a $4 \mathrm{~d}$ chiral spectrum arising from it, the easier way to preserve the $\mathrm{N}=2$ structure is to impose that the integral of any relevant flux vanishes over $\Sigma_{H}$. Note that in supersymmetric SU(5) F-theory models this option is usually not considered, since in order to achieve doublet-triplet splitting a net hypercharge flux is required to thread the Higgs curve(s). However, as mentioned above in the present scheme we are not constrained by the amount of Higgs triplets at the scale $M_{c}$, and one may indeed consider the case where $\int_{\Sigma_{H}} F_{Y}=0$.

In that case both $H_{u}$ and $H_{d}$ arise from the same curve $\Sigma_{H}$, and one may easily implement the mass structure (4.3). Just like for type IIA non-SUSY models, the term $m_{3}^{2} H_{u} H_{d}^{*}+$ c.c. arises by inducing a non-vanishing D-term on this sector, which in this case is induced by worldvolume fluxes on $S$ which do not satisfy the condition $F \wedge J=0, J$ being the Kähler form on $S$. For instance, let $\omega$ be the complex coordinate of $S$ along $\Sigma_{H}$ and $\omega_{\perp}$ the one transverse to it. Then if the flux felt by the doublets in $\Sigma_{H}$ is of the form $F=M_{\|} d \omega \wedge d \bar{\omega}+M_{\perp} d \omega_{\perp} \wedge d \bar{\omega}_{\perp}$ the D-term condition reads $M_{\|}+M_{\perp}=0[65,66]$. Hence, the off-diagonal terms in (4.3) read $m_{3}^{2}=M_{\|}+M_{\perp}$ and arise whenever such vanishing D-term condition is not met.

Finally, the diagonal terms of the mass matrix (4.3) will correspond to a $\mu$-term. In a D7-brane setup dual to the toroidal models of appendix A this mass term appears by simply switching on a continuous or discrete Wilson line along $\Sigma_{H}$. However, as mentioned before Wilson lines are typically not available in F-theory GUT models, and so the $\mu$-term cannot be generated by this mechanism. Instead, such supersymmetric mass term can be induced by the presence of closed string fluxes (see below) or at the non-perturbative level. Indeed, a $\mu$-term may appear at the non-perturbative level from string instanton effects [67-69] (see [35, 70, 71] for reviews). Such $\mu$-terms are automatically symmetric under $H_{u}-H_{d}$ exchange and hence respect the above struc- 
ture. As we said, stringy instanton effects are of order $\exp \left(2 \pi / g_{s}\right)$ and for $g_{s} \simeq 1 / 2$ could give rise to $\mu$-terms of the appropriate order of magnitude $10^{-5} M_{s}$.

ii) SUSY breaking terms from closed string fluxes and modulus dominance

Mass terms for scalar fields may also appear in the presence of closed string fluxes. Indeed this may be explicitly checked by plugging such backgrounds in the DBI+CS action for 7-branes, see [50-56]. In fact it is known that SUSY breaking imaginary self-dual (ISD) IIB 3-form fluxes correspond to giving a non-zero vacuum expectation value to the auxiliary fields of Kahler moduli [72]. So in order to see the effect of closed string fluxes we will work here with the effective action and plug non-vanishing vevs for these auxiliary fields.

In particular, in the context of Type IIB/F-theory compactifications a prominent role is played by the local Kahler modulus $T$ which is coupling to the $\mathrm{SU}(5)$ stack of 7-branes. In a general Type IIB/F-theory compactification this Kahler modulus is the one among a number of such moduli which is relevant for the SUSY breaking soft terms, which will appear when $F_{T} \neq 0$. A good model for this structure is considering the CY manifold $\mathbf{P}_{[1,1,1,6,9]}^{4}$ in refs. [73, 74] with one small Kahler modulus $T$ and one big Kahler modulus $T_{b}$ with Kahler potential

$$
K=-2 \log \left(t_{b}^{3 / 2}-t^{3 / 2}\right) .
$$

with $t=2 \operatorname{Re} T$ and $t_{b}=2 R e T_{b}$. Here one takes $t_{b} \gg t$ and take both large so that the supergravity approximation is still valid. In the F-theory context the analogue of these moduli $t, t_{b}$ would correspond to the size of the 4 -fold $S$ and the 6 -fold $B_{3}$ respectively. For chiral matter fields living at F-theory matter curves one expects a behavior for the Kahler metrics in the dilute flux limit [75]

$$
K=\frac{t^{1 / 2}}{t_{b}}
$$

On the other hand if the Higgs doublets $H_{u}, H_{d}$ in matter curves are not chiral, they behave like scalars in a $N=2$ hypermultiplet, very much like in the previous case of open string fluxes. Under these conditions one expects kinetic terms for the Higgs multiplets of the form

$$
\frac{t^{1 / 2}}{t_{b}}\left|H_{u}+H_{d}^{*}\right|^{2}
$$

This type of Higgs kinetic terms proportional to $\left|H_{u}+H_{d}^{*}\right|^{2}$ have been discussed in the past in the context of heterotic orbifold compactifications with $N=2$ subsectors in the untwisted spectrum and they display a shift symmetry under $H_{u} \rightarrow H_{u}+c, H_{d} \rightarrow$ $H_{d}-c *[77,78]$. Heterotic Type I S-duality indicates that such structure should also be present in Type IIB orientifolds. Recently Hebecker, Knochel and Weigand [79] have proposed that this shift symmetry may be at the origin of the $\tan \beta=1$ boundary condition and studied its appearance also in Type II vacua. In our context the assumption of T-modulus dominance SUSY breaking allows to explicitly compute 
the relevant soft terms. Indeed applying standard supergavity formulae [80] one obtains for the Higgs mass parameters

$$
m_{H_{u}}^{2}=m_{H_{d}}^{2}=\frac{M^{2}}{2} ; \quad \mu=-\frac{M}{2} ; \quad m_{3}^{2}=\frac{3}{4} M^{2}
$$

so that

$$
m_{H_{u}}^{2}+\mu^{2}=m_{H_{d}}^{2}+\mu^{2}=m_{3}^{2}=\frac{3}{4} M^{2}
$$

where $M=F_{T} / t$ is the gaugino mass, with $F_{T}$ the auxiliary field in the $T$ chiral multiplet. Now, unlike the open string flux case, the diagonal masses have both a SUSY contribution and a SUSY-breaking contribution and there is automatically a massless Higgs boson. We again obtain $\tan \beta=1$ at the unification scale, this time automatically due to the mentioned shift symmetry. This value is however renormalized, as we point out below.

As a general conclusion, we see that in string theory models in which the Higgs sector corresponds to a $N=2$ subsector with $H_{u}, H_{d}$ sitting in a hypermultiplet (before SUSY breaking), the condition $m_{H_{u}}^{2} \simeq m_{H_{d}}^{2}$ is naturally obtained. In addition off-diagonal $m_{3}^{2}$ terms may be induced both by effects from open and closed string fluxes.

Let us finally comment on possible generalizations of this minimal Higgs structure. A first generalization is starting with $n_{H}$ sets of Higgs particles above $M_{S S}$. In that case minimal landscape fine-tuning will still prefer that only one combination of the $2 n_{H}$ Higgs scalars remains massless. Depending on how the original Higgs multiplets coupled to the different families the resulting Yukawas could inherit an interesting flavor structure. Another possible extension could be to dispose of R-parity in the initial MSSM spectrum since L/B-violating dimension four operators are suppressed due to the large mass of sfermions. In this case the Higgs $H_{d}$ could mix with sleptons $L_{i}$. However in this case the approximate symmetry under the exchange of $H_{u}$ and $H_{d}$ would typically be absent and the prediction $\tan \beta \simeq 1$ would be in danger, so this particular bilinear should be slightly supressed.

\section{$5 \quad$ Higgs mass fine-tuning}

We have seen how one may naturally obtain a massless Higgs with $\tan \beta=1$ in string theory and, in particular, also in the context of Type IIB constructions with mass terms induced by open and closed string fluxes. In general one has to fine-tune the parameter $m_{3}^{2}$ with the Higgs masses $m_{H_{u}}^{2}=m_{H_{d}}^{2}$. This may be done e.g. by partially canceling the contributions to $m_{3}^{2}$ from open and closed string fluxes.

However the above results are subject to loop corrections which will force to a redefinition of the fine-tuning. If the SUSY breaking scale is of order $M_{S S} \simeq 10^{11} \mathrm{GeV}$ the fine-tuning should be done to at least 4-loop order to cope with a hierarchy of nine orders of magnitude down to the EW scale. Still the idea is that even after these further fine-tuning corrections the Higgs scalar which remains light is approximately the one corresponding to the combination $H_{u}-H_{d}^{*}$, i.e. that approximately $\tan \beta \simeq 1$. 
In fact, if we assume that $\tan \beta\left(M_{S S}\right)=1$ (as e.g. in eqs. (4.13)) before any loop correction is included, we know that the running of the parameters in between the scales $M_{c}$ and $M_{S S}$ will renormalize $\tan \beta$. We expect that the large top quark Yukawa coupling will make $m_{H_{u}}^{2}<m_{H_{d}}^{2}$ after loop corrections. We also expect to obtain one massive Higgs eigenstate and a second one slightly tachyonic. This may be compensated to get a massless Higgs boson at this level by tuning with an open or closed string flux as explained in the previous section. Still, after this fine-tuning, the value of $\tan \beta$ is no longer 1 but is given by $\tan \beta=\left|m_{H_{d}}\left(M_{S S}\right)\right| /\left|m_{H_{u}}\left(M_{S S}\right)\right|$, as explained in the previous section. In addition to this there will be higher order finite loop corrections which are expected to yield smaller negligible contributions to $\tan \beta$. So a good estimation of $\tan \beta$ at the scale $M_{S S}$ should be given by taking into account the running of the parameters in between $M_{c}$ and $M_{S S}$.

To compute the value of $\tan \beta$ at $M_{S S}$ we have to consider the RGE for the MSSM parameters in the region $M_{S S}-M_{c}$. In the present case we know that with a single Higgs field at the electroweak scale only the top-quark Yukawa coupling $h_{t}$ is relevant in this equation. Fortunately, the one-loop RGE in the $h_{t} \gg h_{b}, h_{\tau}$ limit were solved analytically in ref. [81] for the case of universal soft terms, i.e. as in the CMSSM model, which should be more than enough to evaluate this renormalization effect. One has $\tan \beta\left(M_{S S}\right)=\left|m_{H_{d}}\left(M_{S S}\right)\right| /\left|m_{H_{u}}\left(M_{S S}\right)\right|$ with

$$
\begin{aligned}
& m_{H_{d}}^{2}(t)=m^{2}+\mu^{2} q^{2}(t)+M^{2} g(t) \\
& m_{H_{u}}^{2}(t)=m^{2}\left(h(t)-k(t) A^{2}\right)+\mu^{2} q^{2}(t)+M^{2} e(t)+A m M f(t)
\end{aligned}
$$

where $m, M, A, \mu$ are the standard universal CMSSM parameters at the unification scale $M_{c}, t=2 \log \left(M_{c} / M_{S S}\right)$ and $q, g, h, k, e, f$ are known functions of the top Yukawa coupling $h_{t}$ and the three SM gauge coupling constants. For completeness these functions are provided in appendix B. Note that in order to compute $\tan \beta\left(M_{S S}\right)$ we do not need to know how $m_{3}^{2}$ runs since its value is fixed by the fine-tuning condition $m_{3}^{4}=m_{H_{u}}^{2} m_{H_{d}}^{2}$ at $M_{S S}$.

These functions involve integrals of coupling constants over the region $M_{S S}$ to $M_{c}$. There is an explicit dependence on the universal soft terms $m, M, A, B, \mu$ which are all of order $M_{S S}$ but the results are quite insensitive to the precise values of those parameters. For definiteness we have computed $\tan \beta\left(M_{S S}\right)$ for the boundary conditions $m^{2}=M^{2} / 2$, $A=-3 M / 2=B, \mu=-M / 2$, with $M=M_{S S}$, corresponding to the modulus dominance SUSY-breaking soft terms described around eq. (4.12), see e.g. [76]. In figure 5 we show the dependence of $\tan \beta\left(M_{S S}\right)$ as a function of $M_{S S}$, where $M c$ is taken as in section 3 , from the gauge coupling unification condition. One observes that for $M_{S S}$ in the range $10^{8}-10^{12} \mathrm{GeV} \tan \beta$ is only slightly increased to a value around $\tan \beta \simeq 1.2-1.4$, depending on the value of the top-quark mass. In fact using the above formulae one can expand in a power series of the square of the top Yukawa coupling to find

$$
\tan \beta\left(M_{S S}\right)=1.00+h_{t}^{2}\left(M_{c}\right) \times 0.58+\ldots .
$$

It seems then that the tree level value of $\tan \beta$ is only slightly deformed away from 1 after loop corrections. As we said, higher loop effects required to do a fully consistent finetuning are not expected to spoil this conclusion. An analogous conclusion was reached 


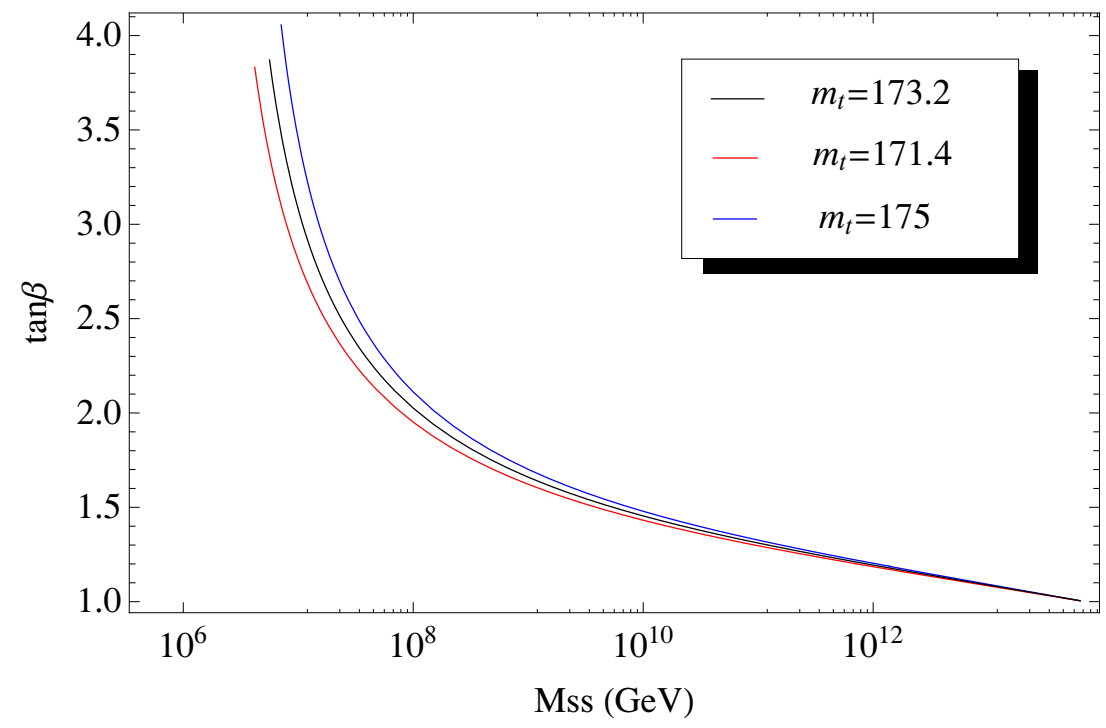

Figure 5. Renormalization of $\tan \beta$ in the region $M_{S S}-M_{c}$ as a function of $M_{S S}$ for three values of the top quark mass.

in [79] using different methods. ${ }^{9}$ One can trivially extend the calculation to the case in which color triplets $D, \bar{D}$ remain below $M_{c}$ with very similar results.

A natural question is whether the Higgs mass terms discussed above scan in the string landscape. These masses depend on the local value of string flux densities in the region in the compact dimensions where the SM fields are localized. These local densities are in general not quantized, it is their integrals over 3-and 2-cycles which are quantized. As is well known in a generic compactification there may be of order a hundred different quantized closed string fluxes which may be turned on. All of them in general may contribute to the cosmological constant and could play a role in its anthropic solution [82]. The required energy spacing for the c.c. constant is so minute that at least some of these fluxes should e.g. be combined with anti-D3-branes on CY-throats in order to be able to fine-tune the c.c. following the KKLT approach [83]. On the other hand only a selected number of fluxes affect the SM branes in the cycle $S$. Again although these fluxes are quantized it is the density $G_{3}$ at the location of the 7-branes which is relevant. However varying the flux quanta one can also control this local density. So, indeed, it seems plausible that the subset of the fluxes going through $S$ will scan in the string landscape. It would be interesting to materialize in some detail this expectation.

\section{Proton decay}

As we already advanced with a unification scale as low as $M_{c}=3 \times 10^{14} \mathrm{GeV}$ there is a danger of dimension 6 operators giving rise to proton decay rates much faster than

\footnotetext{
${ }^{9}$ These results remain unchanged in the case of a R-parity violating MSSM since the new B/L-violating couplings will only appear in the Higgs mass running at two loops.
} 
experiment. In standard field theory GUTs, the proton decay dim=6 operators obtained after integrating out the massive $X, Y$ doublet of gauge bosons are [61]

$$
\begin{aligned}
O_{1} & =\frac{4 \pi \alpha_{G}}{2 M_{X, Y}^{2}} \overline{U_{a L}^{c}} \gamma^{\mu} Q_{a L} \overline{E_{b L}^{c}} \gamma_{\mu} Q_{b L} \\
O_{2} & =\frac{4 \pi \alpha_{G}}{2 M_{X, Y}^{2}} \overline{U_{a L}^{c}} \gamma^{\mu} Q_{a L} \overline{D_{b L}^{c}} \gamma_{\mu} L_{b L} .
\end{aligned}
$$

The first operator arises from the exchange of the heavy gauge bosons with masses $M_{X, Y}$ between two 10-plets whereas the second from the exchange between a 10-plet and a 5-plet. Experimentally, the Super-Kamiokande limit on the chanel $p \rightarrow \pi^{0} e^{+}$gives an absolute lower limit $\tau_{p}>5 \times 10^{33}$ years [84]. This corresponds to a bound on $M_{X, Y}$

$$
M_{X, Y} \geq \sqrt{\frac{\alpha_{G}}{1 / 39}} 1.6 \times 10^{15} \mathrm{GeV}
$$

A value $M_{X, Y}=M_{c}=3 \times 10^{14} \mathrm{GeV}$ is 5 times smaller and that could pose a problem. In F-theory GUTs the same proton decay operators as above will appear, the difference now being that the symmetry is broken due to a hypercharge flux. Due to this fact the coefficients of the operators may change substantially, as we now discuss.

Indeed, considering proton decay in the context of F-theory SU(5) unification provides a new interesting mechanism to suppress proton decay. A microscopic computation of the above dimension 6 proton decay operator would involve first computing couplings of the form e.g. $\overline{U_{a L}^{c}} X_{\mu} Q_{a L}$ and then integrating out the massive doublet $X, Y$. The computation of such trilinear couplings is rather similar to the computation of Yukawa couplings, in the sense that it also involves a triple overlap of internal wavefunctions, namely

$$
\Gamma_{1}^{i j}=\int_{S}\left(\Psi_{10}^{i}\right)^{*} \Psi_{10}^{j} \Phi_{X, Y} \quad \Gamma_{2}^{i j}=\int_{S}\left(\Psi_{5}^{i}\right)^{*} \Psi_{5}^{j} \Phi_{X, Y}
$$

where now $\Phi_{X, Y}$ are the internal wavefunctions of the broken $\mathrm{SU}(5)$ bosons $X, Y$. These form a doublet of massive gauge bosons with quantum numbers $(3,2,5 / 6)+$ c.c. .

In standard 4d GUTs, the value of such couplings does not depend on the vev of the Higgs in the $\mathbf{2 4}$ of $\mathrm{SU}(5)$, and so it is exactly the same before and after $\mathrm{SU}(5)$ breaking (to leading order). Hence, one may extract the trilinear couplings like $\overline{U_{a L}^{c}} X_{\mu} Q_{a L}$ directly from the SU(5) Lagrangian as the strength by which $\mathrm{SU}(5)$ gauge bosons couple to chiral matter, namely $\left(4 \pi \alpha_{G}\right)^{1 / 2}$.

Now, the key point for proton decay suppression in F-theory is the fact that the ingredient that triggers $\mathrm{SU}(5)$ breaking is not a vev for a scalar in the adjoint of $\mathrm{SU}(5)$, but the presence of the hypercharge flux $F_{Y}$ along the GUT 4-cycle $S$. The mass of the $X, Y$ gauge bosons is given by

$$
M_{X, Y}^{2}=\frac{5 N_{Y}}{12 \pi}
$$

where $N_{Y}$ is the flux density of hypercharge flux $F_{Y}$, which we take constant for simplicity. The flux quantization condition implies $5 / 6\left(F_{Y} / 2 \pi\right)$ is quantized in $S$ (i.e., its integral over any 2-cycle of $S$ is an integer), so that $N_{Y} \approx 12 \pi / 5 \mathrm{Vol}_{S}^{-1 / 2}$ and indeed $M_{X, Y} \simeq M_{c} \simeq$ 
$\mathrm{Vol}^{-1 / 4}$. Finding the wavefunctions in (6.4) involves solving a Dirac or Laplace equation for them, in which any flux threading $S$ will enter. We then have that both the wavefunctions for chiral fields and massive gauge bosons $X, Y$ depend on the internal fluxes on $S$, and in particular on the the hypercharge flux $F_{Y}$. As a result, adding an hypercharge flux will necessarily change the value of the effective $4 \mathrm{~d}$ couplings (6.4): while in the absence of $F_{Y}$ such couplings must be $\propto \alpha_{G}^{1 / 2}$ in its presence they will have a new value.

To show that this new value will be suppressed with respect to $\alpha_{G}^{1 / 2}$ we need some machinery from wavefunction computation in F-theory GUT models. Here we will try to be schematic, referring the reader to appendix $\mathrm{C}$ and to [85] (see also [65, 66, 86-92]) for more details on the subject. In F-theory SU(5) models there are basically two kinds of wavefunctions: the ones that are peaked at the matter curves of $S$, namely $\Psi_{10}^{i}, \Psi_{5}^{j}$ and $\Phi_{H_{U, D}}$, and the ones that are spread all over the 4-cycle $S$, namely the $\mathrm{SU}(5)$ gauge bosons and in particular $\Phi_{X, Y}$. As they come from different sectors of the theory, these two kinds of wavefunctions feel the effect of the hypercharge flux in a different way.

Indeed, let us consider the wavefunctions involved in the coupling $\Gamma_{1}$ in (6.4). Solving for them in a local patch of $S$ and assuming that the 4-cycle $S$ is sufficiently large (see appendix $\mathrm{C}$ and [85] for more details) we have that

$$
\begin{aligned}
\Psi_{10}^{i}(x, y) & =\gamma_{10}^{i} m_{*}^{4-i} x^{3-i} e^{-\frac{M}{2}|x|^{2}} e^{-m^{2}|y|^{2}-q_{S} \operatorname{Re}(x \bar{y})} \\
\Phi_{X, Y}(x, y) & =\gamma_{X, Y} m_{*} e^{-\frac{5}{24} N_{Y}\left(|x|^{2}+|y|^{2}\right)}
\end{aligned}
$$

where $(x, y)$ stand for local complex coordinates of the 4-cycle $S$, and we have assumed that matter curve supporting the chiral fields $\mathbf{1 0}$ is given by $\Sigma_{10}=\{y=0\}$. Here $M$ and $N_{Y}$ stand for densities of fluxes threading the 4-cycle $S$. More precisely $N_{Y}$ is the density of the hypercharge flux $F_{Y}$, while $M$ is the density of the flux necessary to have three families of 10's along $\Sigma_{10}$. The hypercharge dependence of the wavefunction $\Psi_{10}$ is encoded in the parameter $q_{S}$, so that for a non-vanishing $F_{Y}$ particles with different hypercharge have different wavefunctions. The parameter $m^{2}$ stands for the slope of the intersection between the SU(5) 4-cycle $S$ and the U(1) 7-brane intersecting $S$ in $\Sigma_{10}$. Such intersection scale is typically of the order of the fundamental scale of F-theory $m_{*}\left(\simeq M_{s}\right.$ in a perturbative IIB orientifold) and much larger than any flux density like $N_{Y}$ or $M$, showing that the $\Psi_{10}^{i}$ are highly peaked along the matter curve $\Sigma_{10}=\{y=0\}$. All of these quantities that appear in the exponential factor of $\Psi_{10}^{i}$ are family independent, the only dependence of the family index $i$ corresponding to the power of $x$ (the matter curve $\Sigma_{10}$ coordinate) that appears in the wavefunction. It has been found [65, 66, 86-91] that with this prescription (that assigns the power $x^{2}$ to the first family, etc.) one can reproduce the mass hierarchy between families observed in nature. Finally, the $\gamma$ 's are normalization factors that insure that such fields are canonically normalized.

Notice that the fact that $N_{Y}, M$ and $m^{2}$ are non-zero gives a gaussian profile to these wavefuctions, and this allows to carry the integral for $\Gamma_{1}$ by replacing $S$ with $\mathbb{R}^{4}$. This is important since otherwise we would need geometrical information about the full manifold $B_{3}$, which is in general not available. Notice also that the wavefunction for the boson $X, Y$ is only affected by the hypercharge density flux $N_{Y}$, and that in the limit $N_{Y} \rightarrow 0$ we recover 
a constant wavefunction. This is to be expected, since at this limit the $\mathrm{SU}(5)$ symmetry is restored and $X, Y$ become massless gauge bosons, which always have a constant profile.

Given these facts we are now ready to compute the coupling $\Gamma_{1}$ above. First notice that in the limit $N_{Y} \rightarrow 0$ the integral is trivial in the sense that $\Phi_{X, Y}=\gamma_{X, Y} m_{*}$ is constant, since

$$
m_{*} \int_{S}\left(\Psi_{10}^{i}\right)^{*} \Psi_{10}^{j} \Phi_{X, Y}=\gamma_{X, Y} m_{*}^{2} \int_{S}\left(\Psi_{10}^{i}\right)^{*} \Psi_{10}^{j} \approx \alpha_{G}^{1 / 2} \delta^{i j}
$$

where have added the correct factor of $m_{*}$ and used that for $N_{Y}=0$, the normalization factor is simply $\gamma_{X, Y}=\operatorname{Vol}_{S}^{-1 / 2} m_{*}^{-2} \approx \alpha_{G}^{1 / 2}$. Hence in this limit we recover the result expected from $\mathrm{SU}(5)$ gauge invariance.

This result is no longer true when $N_{Y} \neq 0$ and the wavefunction $\Phi_{X, Y}$ has a nontrivial profile. Then one finds that there is a suppression in the above coupling which is family dependent, and bigger for lower families. Indeed, to get an estimate of this coupling it is useful to use the fact that $m^{2} \sim m_{*}^{2} \gg M, N_{Y}$ and treat the Gaussian profile $\exp \left(-m^{2}|y|^{2}\right)$ as a $\delta$-function in the coordinate $y$, which is nothing but asking that the matter wavefunctions $\Psi_{10}^{i}$ are fully localized in $\Sigma_{10}$. That is, we take the limit $m^{2} \rightarrow \infty$ in which

$$
\left(\Psi_{10}^{i}\right)^{*} \Psi_{10}^{j} \rightarrow \gamma_{10}^{i} \gamma_{10}^{j} m_{*}^{8-i-j} \bar{x}^{3-i} x^{3-j} e^{-M|x|^{2}} \delta(y)
$$

and so the integral must be basically taken over $\Sigma_{10}$. Taking into account that in this limit the normalization factors are

$$
\gamma_{10}^{i}=\frac{1}{\sqrt{(3-i) \pi}}\left(\frac{M}{m_{*}^{2}}\right)^{\frac{4-i}{2}} \quad \gamma_{X, Y}=\frac{5 N_{Y}}{12 \pi m_{*}^{2}}
$$

we obtain that

$$
m_{*} \int_{S}\left(\Psi_{10}^{i}\right)^{*} \Psi_{10}^{j} \Phi_{X, Y}=\delta^{i j} \frac{5 N_{Y}}{12 \pi m_{*}^{2}}\left(\frac{M}{M+\frac{5}{24} N_{Y}}\right)^{4-i} \approx \delta_{i j} \alpha_{G}^{1 / 2}\left(\frac{M}{M+\frac{5}{24} N_{Y}}\right)^{4-i}
$$

where we have used again $N_{Y} \approx 12 \pi / 5 \mathrm{Vol}_{S}^{-1 / 2}$. This result is reproduced in appendix C without taking the $\delta$-function approximation.

Notice that the coupling (6.11) is indeed suppressed with respect to the 4d GUT result $\alpha_{G}^{1 / 2}$, and that the suppression is bigger the lighter the family. Since we are interested in proton decay operators one could in principle focus on the first family $i=1$, in which by assuming that the densities $M$ and $5 / 24 N_{Y}$ are equal we already obtain a suppression factor of $1 / 8$, and much bigger if $5 / 24 N_{Y}>M$. In fact, being more rigorous, we would really need to take into account the fact that the actual physical first generation wave functions will be proportional to a linear combination of the $x^{2}, x, 1$ monomials. Even if this extra terms are present, one expects the first generation to be dominated by the $x^{2}$ monomial with a small contamination (related to mixing angles) from the other two. ${ }^{10} \mathrm{In}$ any event, the presence of a suppression will be generic.

The fact that the suppression factor is bigger for each family can be given an intuitive understanding, since in F-theory families with smaller Yukawa couplings are those that have

\footnotetext{
${ }^{10}$ We thank P. Cámara for discussions on these points.
} 


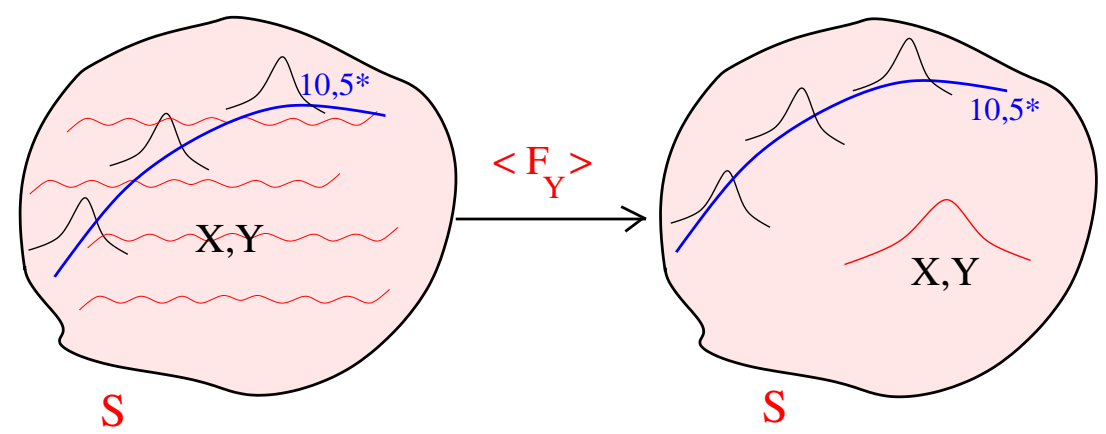

Figure 6. Coupling of $\mathrm{SU}(5)$ off-diagonal gauge bosons $X, Y$. Before symmetry breaking by hypercharge fluxes the wave function of $X, Y$ is extended over the whole 4-cycle $S$. After the hypercharge flux $F_{Y}$ in introduced their wavefunction is localized and their coupling to $10, \overline{5}$ fields is supressed.

a higher polynomial degree $x^{n}$ in their wavefunction (see eq. (6.6)). Such higher power gives a compensating effect to the localization that arises from the family independent exponential factor $\exp \left(-a|x|^{2}\right)$, that tends to localize the triple overlap around $x=0$. The lighter the family the bigger the compensating effect, thus the smaller the coupling.

This understanding of the coupling strength in terms of exponential factors gives yet another mechanism for suppressing the dimension six proton decay operators. Indeed, notice that in (6.7) we have described the wavefunction for the massive $X, Y$ bosons in terms of a Gaussian function on $S$ peaked at $x=y=0$. However, that the wavefunction $\Phi_{X, Y}$ peaks there is in fact a choice that we have made biased by the local patch description of our F-theory model setup. Unlike for the wavefunctions $\Psi_{10}^{j}$, whose equations of motion force them to be localized at the matter curve $\Sigma_{10}=\{y=0\}$, there is nothing special about $y=0$ for the wavefunctions of the gauge bosons $X, Y$ which only depends on the hypercharge flux $F_{Y}$ and on the geometry of the 4-cycle $S$. Only these two factors will determine where the peak of the wavefunction $\Phi_{X, Y}$ is, so there is a priory no reason to think that it will be peaked at any matter curve. Now, if the wavefunction $\Phi_{X, Y}$ is not peaked at $y=0$ but somewhere else the $\delta$-function in (6.9) will yield an extra suppression upon integration on the complex coordinate $y$, as the wavefunction density for $\Phi_{X, Y}$ will be exponentially suppressed away from its peak.

To summarize, F-theory SU(5) models have naturally suppressed dimension 6 proton decay operators, because the mechanism that breaks the SU(5) symmetry - the hypercharge flux $F_{Y}$ - also affects the couplings where these operators come from. Indeed, the presence of the hypercharge flux deforms the wavefunction profile for the fields $\mathbf{1 0}, \overline{\mathbf{5}}$ and $X, Y$, as illustrated in figure 6 . In particular it affects the $X, Y$ bosons, which instead of being massless gauge bosons extended evenly over the whole 4-cycle $S$, are due to $F_{Y}$ massive modes peaked at some point of it. Such localization effect indeed changes the value of the couplings (6.4) as we have shown above in a simplified computation reproduced in more detail in appendix C. Moreover, for the sake of simplicity we assumed above that the peak of the $X, Y$ wavefunction lied on top of the matter curve $\Sigma_{10}$ where the 10 -plet resides. There 
is no reason for this assumption to hold in a global description of our setup, so the $X, Y$ wavefunction will in general be suppressed in the region of $\Sigma_{10}$ and there will be a further suppression to the coupling of $X, Y$ to quarks-leptons. It is easy to see that any of these suppression mechanisms allow to have a rate for proton decay consistent with experimental limits. Note however that the precise value of the coefficient of the operators depends on the details (i.e. local fluxes) of the model. Still these results allow for the possible detection of proton decay through e.g. the channel $p \rightarrow \pi^{0} e^{+}$, typical of non-SUSY unification, in future proton decay experiments.

If Higgs triplets $D, \bar{D}$ with a mass $M_{D} \simeq M_{S S} \simeq 10^{11} \mathrm{GeV}$ are present in the spectrum, there will appear additional contributions to proton decay close to the present experimental limits [61]. They would come from the exchange of the scalar fields $\tilde{D}, \tilde{\bar{D}}$ among quarks and leptons of the first and second generations from Yukawa couplings, with $p \rightarrow \mu^{+} K^{0}$ the dominant channel. In field theory GUTs these Yukawa couplings are directly related to the Yukawa couplings of the Higgs doublets due to the SU(5) symmetry. In our case however the relevant D-field Yukawas are different to those of the Higgs, again due to the presence of the hypercharge flux [85]. One still expects those Yukawas to be of the same order of magnitude, i.e. of order $10^{-5}$ for the first generation. The combination of a massive D-field with the smallness of Yukawa couplings make these extra dimension 6 contributions compatible with experimental bounds, given the uncertainties.

Note in closing that dimension 5 proton decay operators are very much suppressed in the present framework due to the large mass of the SUSY partners. Additional sources of proton decay could appear if the underlying MSSM contains dimension 4 R-parity violating couplings. These could give rise to new dimension 6 operators by the exchange of sfermions but the rate will be again suppressed by the large mass of the SUSY partners combined with the expected smallness of the R-parity violating couplings involving the first generations.

\section{Other consequences}

\subsection{Axions}

The strong CP problem is a naturality problem with no obvious anthropic solution. In this sense it is quite satisfactory that string theory has natural candidates for the axion solution of the strong CP problem. As shown in eq. (3.1) the imaginary part of the local Kähler modulus Im $T$ has axionic couplings to the QCD gauge bosons, and hence is in principle an axion candidate which could solve the strong CP problem. ${ }^{11}$ In the Type IIB/F-theory scheme under discussion it is an important point the decoupling of the local GUT physics sitting on the local $S$ 4-cycle from the global physics of the full six extra dimensions. In particular it is the local Kahler modulus $T$ which couples to the $\mathrm{SU}(5)$ gauge bosons as shown in eq. (3.1). One can compute the associated axion scale $F_{a}$ from the kinetic term

\footnotetext{
${ }^{11}$ The $\tau$ complex dilaton scalar has also axionic couplings but $\operatorname{Im} \tau$ gets generically massive in the presence of closed string fluxes.
} 
of the modulus $T$ (see e.g. [35])

$$
F_{a}^{2}=\frac{M_{p}^{2}}{4 \pi\left(8 \pi^{2}\right)^{2}} \frac{\partial K\left(T, T^{*}\right)}{\partial T \partial T^{*}}=\frac{M_{p}^{2}}{4 \pi\left(8 \pi^{2}\right)^{2}} \frac{3 t^{-1 / 2}}{8 t_{b}^{3 / 2}}
$$

where in the last equality we have used eq. (4.9), which correctly features the decoupling of the local SU(5) physics from the global properties of the compact manifold. For the local modulus one has $t=1 / \alpha_{G}$ and

$$
t_{b}=\frac{V_{6}^{2 / 3}}{g_{s} \alpha^{\prime 2}(2 \pi)^{4}}=\frac{\alpha^{\prime 1 / 2} g_{s}^{1 / 4}}{\sqrt{8}} M_{p}
$$

where in the last equality we have used eq. (2.1). Using eq. (2.3) one finally obtains

$$
F_{a}=\left(\frac{18}{\pi^{2}}\right)^{1 / 4} \frac{M_{c}}{16 \pi^{2}}
$$

Note that the axion decay constant is directly related to the compactification scale (or the string scale via eq. (2.3)) and hence may be naturally low. This is to be contrasted to the heterotic model-independent axion $\operatorname{Im} S$ whose axionic coupling is directly tied to the Planck scale through $F_{a}^{\text {het }}=\alpha_{G} M_{p} /\left(8 \pi^{3 / 2}\right) \simeq 10^{16} \mathrm{GeV}$ (see e.g. [35] and references therein). In our case, for the preferred value $M_{c}=3 \times 10^{14} \mathrm{GeV}$ one obtains

$$
F_{a} \simeq 2 \times 10^{12} \mathrm{GeV}
$$

This is an interesting value since $F_{a}$ it is in the allowed QCD invisible axion range. It is at the upper limit of the allowed window, which is in fact required for the axion to be a viable dark matter candidate. This is also fortunate because in this scheme there are no light neutralinos as in the MSSM or split SUSY which could play the role of dark matter.

The mass of the axion is given through standard formulae by (see. e.g. [93])

$$
m_{a}=\frac{z^{1 / 2}}{(1+z)} \frac{f_{\pi} m_{\pi}}{F_{a}}=\frac{0.6 \times 10^{3} \mu e V}{F_{a} /\left(10^{10} \mathrm{GeV}\right)}
$$

where we have taken $z=m_{u} / m_{d}=0.56$. For the $F_{a}$ value in (7.4) one gets an axion mass

$$
m_{a} \simeq 2.7 \mu \mathrm{eV}
$$

Due to the underlying $\mathrm{SU}(5)$ symmetry the coupling of the axion to photons is directly related to $F_{a}$ by a factor $\sin ^{2} \theta_{W}=3 / 8$ (this is analogous to the DFSZ axion case [94, 95]). In particular, defining the (normalized) axion-photon coupling as

$$
\frac{G_{a \gamma \gamma}}{4} a F^{\gamma} \wedge F^{\gamma}
$$

one obtains

$$
G_{a \gamma \gamma}=\frac{\alpha_{e m}}{2 \pi F_{a}}\left(\frac{8}{3}-\frac{2}{3} \frac{(4+z)}{(1+z)}\right) \simeq 0.38 \times 10^{-15}(G e V)^{-1} .
$$


These values are not far from the limits obtained from searches with the microwave cavity experiment ADMX for cosmic axion dark matter [96]. They obtain

$$
\frac{\left|G_{a \gamma \gamma}\right|}{m_{a} / \mu e V}<5.7 \times 10^{-16}(\mathrm{GeV})^{-1} \sqrt{\frac{0.45 \mathrm{GeV} / \mathrm{cm}^{3}}{\rho_{D M}}}
$$

for $m_{a}$ in a range $m_{a}=1.9-3.55 \mu \mathrm{eV}$. Here $\rho_{D M}$ is the local dark matter density. In our case we have $\left|G_{a \gamma \gamma}\right| /\left(m_{a} / \mu e V\right) \simeq 1.4 \times 10^{-16}\left(\mathrm{GeV}^{-1}\right)$. The upgrading of ADMX should be able to test the axion parameters of the present scheme. ${ }^{12}$ This would be an important test of these ideas.

Let us finally comment that a possible problem for the axion in the local modulus $T$ to become a QCD axion is moduli fixing. Indeed one may wonder whether the dynamics fixing the moduli could also give a large mass to $\operatorname{Im} T$. However this is not necessarily the case see e.g. [98-100].

\subsection{Cosmology}

The main new ingredient in this ISSB scheme is the large SUSY breaking scale $M_{S S} \simeq$ $5 \times 10^{10} \mathrm{GeV}$ and relatively low string scale $M_{s} \simeq 6 \times 10^{14} \mathrm{GeV}$. It is a true fact that having low-energy SUSY leads to a number of problems which are automatically solved with such large SUSY scale. In particular there is no moduli, gravitino nor Polony problem.

Another problem which is solved is the one first pointed out in [101]. This problem appears in string moduli fixing models like KKLT and other extensions in which a supergravity scalar potential combined with other SUSY breaking effects (like antibranes) fix the moduli. Including the inflaton within such schemes leads to the conclusion that the Hubble scale at inflation $H_{I}$ must verify $H_{I}<m_{3 / 2}$ in order for moduli fixing not to be destroyed. In a low scale SUSY model with $m_{3 / 2}<1 \mathrm{TeV}$ that poses a problem. In our case however with $m_{3 / 2} \simeq 5 \times 10^{10} \mathrm{GeV}$ the problem disappears and inflation and KKLT type of moduli fixing are easily compatible. From this point of view one could argue that inflation in models with KKLT-type moduli fixing suggests a SUSY scale $M_{S S}>10^{10} \mathrm{GeV}$.

\subsection{Neutrino masses}

Singlets playing the role of right-handed neutrinos may appear in F-theory GUT schemes. A natural source of masses for right-handed neutrinos in this framework is string instantons see refs. [67-71]. In our case the instanton suppression is typically of order $\exp \left(-2 \pi / g_{s}\right)$ which may be of order $10^{-6}$. Thus one can generate right-handed neutrino masses of order

$$
m_{\nu_{R}} \simeq e^{-2 \pi / g_{s}} \times M_{s} \simeq 10^{-6} M_{s} \simeq 0.7 \times 10^{9} \mathrm{GeV}
$$

for $g_{s}=0.47$ as suggested by the size of gauge threshold corrections. For neutrino Dirac masses in the range $1-10^{-2} \mathrm{GeV}$ this gives left-handed neutrino Majorana masses in the correct ballpark.

If the theory above $M_{S S}$ is an R-parity violating version of the MSSM, there may be additional sources of neutrino masses. In particular if there are R-parity violating terms of

\footnotetext{
${ }^{12}$ See ref. [97] and references therein for recent ideas of about axions in the context of fine-tuning.
} 
the form $M_{S S}\left(L_{i} H_{u}\right)$ the leptons remaining at low-energies mix with the Higgsinos. After the Higgs gets a vev this induces see-saw Majorana neutrino masses of order $M_{W}^{2} / M_{S S}$ which may also be consistent with the observed neutrino masses.

\section{High Scale SUSY versus Split SUSY}

In previous sections we have concentrated in the ISSB case in which the theory below the SUSY breaking scale $M_{S S}$ is just the SM. A different alternative in the literature is that of Split-SUSY $[18,19]$ in which one assumes that below $M_{S S}$ there is the SM plus in addition the gauginos and Higgsinos. The latter then get SUSY-breaking masses in a region close to the EW scale. One can also repeat the analysis in this case. Concerning gauge coupling unification, we just have to replace the $\beta$-function coefficients of the SM by $\left(b_{1}^{s p}, b_{2}^{s p}, b_{3}^{s p}\right)=(45 / 6,-7 / 6,-5)$. One then finds from eq. (3.7)

$$
12 \log \frac{M_{S S}}{M_{E W}}+12 \log \frac{M_{c}}{M_{S S}}=2 \pi\left(\frac{1}{\alpha_{1}\left(M_{E W}\right)}-\frac{1}{\alpha_{2}\left(M_{E W}\right)}-\frac{2}{3 \alpha_{3}\left(M_{E W}\right)}\right),
$$

i.e., the dependence on $M_{S S}$ cancels out. This means one can always choose threshold effects (i.e. value of $g_{s}$ ) such that one-loop unification occurs. One finds the unification scale is fixed at $M_{c} \simeq 3 \times 10^{16} \mathrm{GeV}$, for any $M_{S S}$ (see figure 7). One also obtains $g_{s}=2-9$ as one goes from $M_{S S}=10^{14} \mathrm{GeV}$ to $M_{S S}=1 \mathrm{TeV}$. This means that the threshold corrections are in general small, as expected from the fact that the unification of the MSSM and Split-SUSY work numerically in quite a similar way.

As found e.g. in [27] if the Higgs mass is in the range $124-126 \mathrm{GeV}$, the value of $M_{S S}$ for Split-SUSY is in the region $10^{7}-10^{4} \mathrm{GeV}$ as one goes from $\tan \beta=1$ to $\tan \beta=50$. The renormalization of $\tan \beta$ above $M_{S S}$ works exactly as in section 5 . Repeating the analysis we find that $\tan \beta$ remains close to one for energies above $10^{7} \mathrm{GeV}$. Below those energies the loop corrections become increasingly important and $\tan \beta$ grows sharply close to $100 \mathrm{TeV}$. Although a detailed analysis would be required, we expect that a Higgs mass in the range $124-126 \mathrm{GeV}$ will be compatible with the boundary condition eq. (4.2) for $M_{S S} \simeq 100 \mathrm{TeV}$. Thus, if the present Higgs hints are confirmed, Split-SUSY would be equivalent for all practical purposes to a fine-tuned MSSM with the scalar sparticles heavier than the SUSY fermions.

Note that, unlike the case of High Scale SUSY, such low values for $M_{S S}$ are not generic if SUSY-breaking is induced by closed string fluxes which yield in our case from eq. (2.5) $M_{S S} \simeq 10^{14} \mathrm{GeV}$ (see figure 7 ). With such high value of $M_{S S}$ one expects from [27] a Higgs mass around $140 \mathrm{GeV}$. So in order to be in agreement with a Higgs mass in the region $124-126 \mathrm{GeV}$ the SUSY breaking flux effects should be substantially diluted. In this respect Split-SUSY is not worse than the MSSM which also requires flux suppression to get soft terms of order $M_{S S} \simeq 1 \mathrm{TeV}$. Concerning the axion decay constant, since in this case $M_{c} \simeq 10^{16} \mathrm{GeV}$ one obtains a high value $F_{a} \simeq 10^{14}$ difficult to reconcile with cosmological limits. On the other hand the theory is automatically safe against too fast proton decay through dimension 6 operators due to the large value of the unification scale $M_{c}$ and Higgsinos/Neutralinos may provide for the dark matter. A summary of the scales in Split-SUSY is shown in figure 7. 


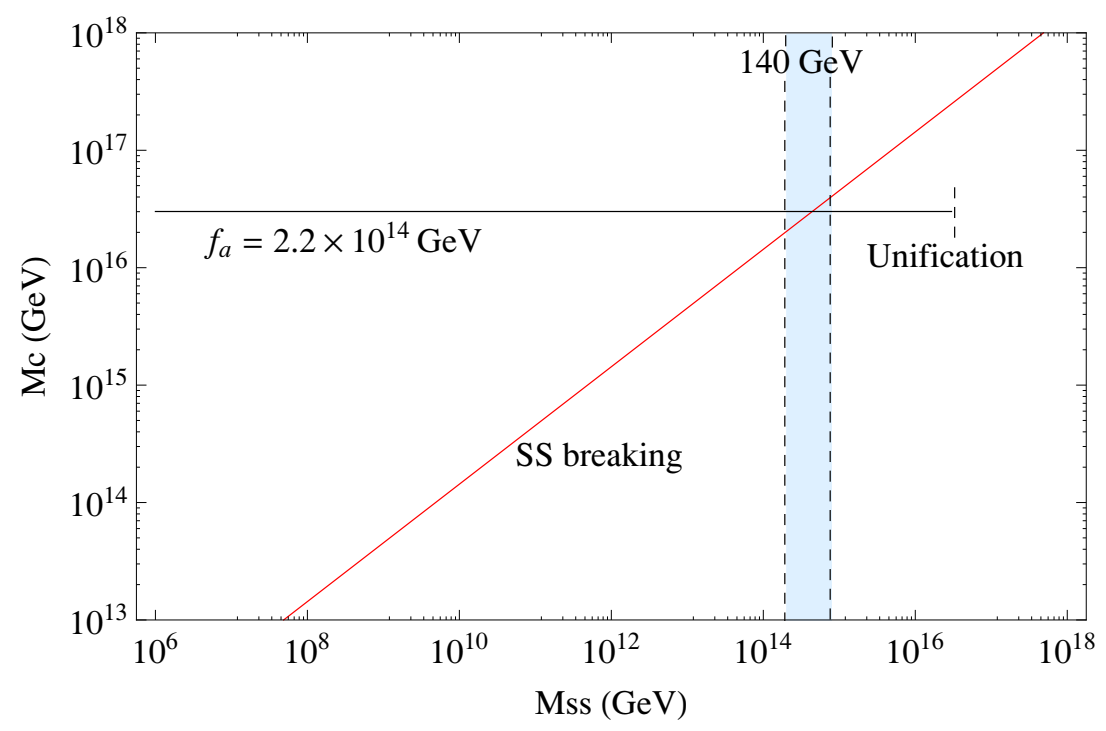

Figure 7. Unification and SUSY breaking scales in Split-SUSY versus the Higgs mass. Flux SUSY breaking naturally prefers $M_{S S} \simeq 10^{14} \mathrm{GeV}$, corresponding to a Higgs mass around $140 \mathrm{GeV}$.

A relevant question is whether one can obtain a Split-SUSY type of spectrum below $M_{S S}$ in string compactifications. We need a first stage of SUSY-breaking in which only the scalars (but the SM Higgs) get a mass of order $M_{S S}$. One simple way to get such masses is through open string fluxes [102-104], very much as in the example in appendix A. A second stage of SUSY-breaking, perhaps from (suppressed) closed string fluxes could give rise to gaugino and Higgsino masses close to $1 \mathrm{TeV}$.

As a summary, High Scale SUSY breaking [20] with $M_{S S} \simeq 10^{11} \mathrm{GeV}$ looks like more natural in the sense that this value of $M_{S S}$ is consistent both with the Higgs mass hints and a generic value of SUSY-breaking effects from fluxes. Furthermore it is simpler in that only one-step of SUSY breaking is needed. ${ }^{13}$ If the Higgs hints are correct, Split-SUSY becomes really a fine-tuned version of the MSSM.

\section{Final conclusions and outlook}

The LHC results are already giving us substantial information on the physics at the electroweak scale. No new physics has been observed as yet and, in particular, SUSY colored particles remain elusive. As the energy and luminosity is increased the experimental constraints imply quite strong conditions on the MSSM parameters which need to be fine-tuned at the per-mil level. If the hints of a Higgs mass around $125 \mathrm{GeV}$ are confirmed this fine-tuning

\footnotetext{
${ }^{13}$ Note also that in Split-SUSY with a scale $M_{S S} \simeq 10^{5}-10^{7} \mathrm{GeV}$, within a SU(5) scheme one needs to have some doublet-triplet splitting mechanism. Furthermore some discrete symmetry like R-parity should also exist to forbid fast proton decay, unless the R-parity violating couplings are extremely suppressed. On the other hand in High Scale SUSY with $M_{S S} \simeq 10^{11} \mathrm{GeV}$ the existence of doublet-triplet splitting or R-parity conservation are not necessary. This is particularly relevant since getting SU(5) vacua with doublet-triplet splitting and R-parity conservation turns out to be non-trivial in string compactifications.
} 
will be strengthened. Such a Higgs mass is a bit too heavy for the MSSM and a bit too low for the SM high energy stability.

In view that some level of fine-tuning is required a natural question is whether after all one should accept a fine-tuning explanation for the hierarchy problem. One could argue that the size of the EW scale could be selected on anthropic grounds, in analogy to Weinberg's anthropic arguments about the cosmological constant [82]. It is certainly premature to give up on the SUSY solution of the hierarchy problem but it makes sense to explore what the alternatives are.

While the fine-tuning idea is worth exploring, it does not imply to give up the supersymmetry idea at some level. We know that supersymmetry is a basic ingredient in string theory and, even if it does not survive at the EW scale, it could have some role at higher energies. In particular in string compactifications non-SUSY theories have generically tachyons in their spectrum and are hence unstable. So it makes sense to investigate whether the theory becomes supersymmetric above some scale $M_{S S}$ in between $M_{E W}$ and the string scale $M_{s}$. Guided by the apparent unification of coupling constants we would also like to know whether this could be combined with some form of unification like $\mathrm{SU}(5)$.

In this paper we address this issue within the context of string theory. In particular we study the possible structure of mass scales in F-theory unified schemes in which a version of $\mathrm{SU}(5)$ unification is possible. We find that there are several pieces of information pointing to a SUSY structure above an intermediate scale $M_{S S} \simeq 5 \times 10^{10} \mathrm{GeV}$ and a unification scale $M_{c} \simeq 3 \times 10^{14} \mathrm{GeV}$. F-theory SU(5) unification differs from field theory unification in some important aspects. The breaking of SU(5) to the SM via hypercharge fluxes generates very specific corrections to the gauge couplings. Combining these corrections with the idea of closed string flux SUSY breaking fixes the scale of SUSY breaking at that intermediate scale. Interestingly, the extrapolation up in energies of the SM Higgs self-coupling $\lambda$ seems to vanish also around that scale, giving additional evidence. This is also consistent with the MSSM-like boundary conditions $\lambda=\frac{1}{8}\left(g_{1}^{2}+g_{2}^{2}\right) \cos ^{2} 2 \beta$ if $\tan \beta \simeq 1$ at $M_{S S}$. We have shown how such value for $\tan \beta=1$ is expected in known sources of SUSY breaking in string theory and is also stable under loop corrections. An additional support for an ISSB structure of scales is obtained from the existence of a natural axion candidate with a decay constant $F_{a} \simeq M_{c} /(4 \pi)^{2} \simeq 2 \times 10^{12} \mathrm{GeV}$, which could solve the strong $\mathrm{CP}$ problem and constitute the observed dark matter.

The unification scale $M_{c} \simeq 3 \times 10^{14} \mathrm{GeV}$ is relatively low and in field theory SU(5) would be ruled due to too fast proton decay via dimension 6 operators. We have shown however that in the context of F-theory SU(5) there is an effect due to the fact that $\mathrm{SU}(5)$ is broken by hypercharge fluxes, not a Higgs mechanism. In this case the hypercharge fluxes modify the profile wave functions of matter and $X, Y$ fields suppressing the couplings which could mediate fast proton decay. If the suppression is not excessive, proton decay e.g. through the standard non-SUSY channel $p \rightarrow \pi^{0} e^{+}$could be measured in future experiments. In the present context doublet-triplet splitting is not unavoidable, the Higgs triplets may remain massless below $M_{c}$ and get masses of order $M_{S S} \simeq 10^{11} \mathrm{GeV}$. They can give additional contributions to proton decay with a dominant $p \rightarrow \mu^{+} K^{0}$ channel. 
If this ISSB framework is correct, no new particles beyond those of the SM and the Higgs particle would be uncovered at the LHC. On the other hand, since the value of $M_{S S}$ within this scheme is fixed by gauge coupling unification + closed string flux breaking, one may consider the vanishing of the Higgs self-coupling at $M_{S S} \simeq 10^{11} \mathrm{GeV}$ as a prediction of this framework. At present the uncertainties on the Higgs and top quark masses do not allow for a definite conclusion. On the other hand more precise measurements of these quantities could confirm or exclude these ideas. Since in the present scheme dark matter is provided by axions, no WIMP's are required at the EW scale, but microwave cavity experiments like the upgraded AMDX experiment, could detect axions in the range here predicted. The observation of proton decay could also provide additional support for this scheme.

\section{Acknowledgments}

We thank P.G. Cámara, A. Font, and A. Uranga for discussions. This work has been partially supported by the grants FPA 2009-09017, FPA 2009-07908, Consolider-CPAN (CSD2007-00042) from the MICINN, HEPHACOS-S2009/ESP1473 from the C.A. de Madrid and the contract "UNILHC" PITN-GA-2009-237920 of the European Commission. F.M. is supported by the Ramón y Cajal programme through the grant RYC-2009-05096 and by the People Programme of FP7 (Marie Curie Auction) through the REA grant agreement PCIG10-GA-2011-304023. D.R. is supported through the FPU grant AP2010-5687.

\section{A Non SUSY SM Type II orientifolds with open string fluxes}

One of the simplest constructions in string theory yielding a SM group with three quark lepton generations is that of toroidal Type IIA orientifolds. In these constructions (see e.g.([35, 105-109]) and references therein) the SM particle are localized at the intersections of D6-branes. In the simplest schemes one has $3+2+1+1$ sets of D6-branes corresponding to a gauge group $\mathrm{U}(3) \times \mathrm{U}(2) \times \mathrm{U}(1) \times \mathrm{U}(1)$. The extra $\mathrm{U}(1)$ 's beyond hypercharge get massive through a Green-Schwarz mechanism and one is left with the gauge group of the standard model. The worldvolume of D6-branes is $4+3$ dimensional with the three extra dimensions being wrapped as 1-cycles in each of the three tori in the $T^{2} \times T^{2} \times T^{2}$ extra dimensions. The number of times the D6-brane wraps the $\left(x^{i}, y^{i}\right), i=1,2,3$ cycles in each of the three tori is parametrized by integer numbers $\left(n_{1}, m_{1}\right) \times\left(n_{2}, m_{2}\right) \times\left(n_{3}, m_{3}\right)$. Chiral fermions and scalars appear at the brane intersections and the multiplicity of generations appear because D6-branes intersect typically a multiple number of times. The number of intersections between two branes $a$ and $b$ is given by the topological invariant

$$
I_{a b}=\left(n_{1}^{a} m_{1}^{b}-m_{1}^{a} n_{1}^{b}\right)\left(n_{2}^{a} m_{2}^{b}-m_{2}^{a} n_{2}^{b}\right)\left(n_{3}^{a} m_{3}^{b}-m_{3}^{a} n_{3}^{b}\right)
$$

The intersection angle between two branes $a, b$ in the $\mathrm{i}$-th torus is given by $\tan ^{-1}\left(m_{i}^{a} U_{i} / n_{i}^{a}\right)-\tan ^{-1}\left(m_{i}^{b} U_{i} / n_{i}^{b}\right)$, where $U_{i}=R_{i}^{y} / R_{i}^{x}$ are the three complex structure parameters of the tori. One can easily find choices of brane wrapping numbers such that the obtained chiral fermions correspond to those of the SM with three generations, see [63]. 


\begin{tabular}{|c||c|c|c|}
\hline$N_{i}$ & $\left(n_{i}^{1}, m_{i}^{1}\right)$ & $\left(n_{i}^{2}, m_{i}^{2}\right)$ & $\left(n_{i}^{3}, m_{i}^{3}\right)$ \\
\hline \hline$N_{a}=3$ & $\left(1 / \beta^{1}, 0\right)$ & $\left(n_{a}^{2}, \epsilon \beta^{2}\right)$ & $(1 / \rho, 1 / 2)$ \\
\hline$N_{b}=2$ & $\left(n_{b}^{1},-\epsilon \beta^{1}\right)$ & $\left(1 / \beta^{2}, 0\right)$ & $(1,3 \rho / 2)$ \\
\hline$N_{c}=1$ & $\left(n_{c}^{1}, 3 \rho \epsilon \beta^{1}\right)$ & $\left(1 / \beta^{2}, 0\right)$ & $(0,1)$ \\
\hline$N_{d}=1$ & $\left(1 / \beta^{1}, 0\right)$ & $\left(n_{d}^{2},-\beta^{2} \epsilon / \rho\right)$ & $(1,3 \rho / 2)$ \\
\hline
\end{tabular}

Table 1. D6-brane wrapping numbers giving rise to a SM spectrum.

In particular there is a large family of 3-generation models of this class which may be obtained from the wrapping numbers in table 1 . Here $N_{i}$ give the number of parallel branes giving rise to a gauge group $\mathrm{U}\left(N_{i}\right)$. Along with each stack of branes there should be additional mirror D6-branes in order for the brane configuration to be invariant under the orientifold operation. Those mirror branes are obtained by flipping the sign of the $m$ component of the wrapping numbers and are not displayed in the table. The general solutions are parametrized by a phase $\epsilon= \pm 1$, the NS background on the first two tori $\beta^{i}=1-b^{i}=1,1 / 2$, four integers $n_{a}^{2}, n_{b}^{1}, n_{c}^{1}, n_{d}^{2}$ and a parameter $\rho=1,1 / 3$. The reader may check that e.g. there are three right-handed leptons at the intersection of branes $\mathrm{c}$ and d, i.e., $I_{c d}=3$ from equation (A.1). We are interested here in the Higgs sector. The Higgs doublets appear from open strings exchanged between the $b$ and $c$ branes (or the mirror brane $\left.c^{*}\right)$. As one can see in table 1 the $\mathrm{U}(2)$ branes $\left(b, b^{*}\right)$ are parallel to the $\left(c, c^{*}\right)$ branes along the second torus and hence they do not intersect. However there are open strings which stretch in between both sets of branes and which lead to light scalars when the distance $Z_{2}$ in the second torus is small. In particular there are Higgs doublets $h_{u}, h_{d}$ from $(b c)$ intersections and $H_{u}, H_{d}$ from $\left(b c^{*}\right)$ intersections, although for some choices of wrapping numbers only the $h$ 's or the $H$ 's survive. In particular there are the scalar states

$$
\begin{aligned}
& \text { State } \\
& \left(-1+\vartheta_{1}, 0, \vartheta_{3}, 0\right) \alpha^{\prime}(\mathrm{Mass})^{2}=\frac{Z_{2}}{4 \pi^{2}}+\frac{1}{2}\left(\vartheta_{3}-\vartheta_{1}\right) \\
& \left(\vartheta_{1}, 0,-1+\vartheta_{3}, 0\right) \alpha^{\prime}(\mathrm{Mass})^{2}=\frac{Z_{2}}{4 \pi^{2}}+\frac{1}{2}\left(\vartheta_{1}-\vartheta_{3}\right)
\end{aligned}
$$

where $Z_{2}$ is the distance ${ }^{2}$ (in $\alpha^{\prime}$ units) in transverse space along the second torus. $\vartheta_{1}$ and $\vartheta_{3}$ are the relative angles between the $b$ and $c$ (or $b$ and $c^{*}$ ) in the first and third complex planes. The states are defined above as vectors in the $\mathrm{SO}(8)$ light-cone target space of Type IIA string theory [35]. There are fermionic states also of the form

$$
\begin{array}{cc}
\text { State } & \text { Mass }^{2} \\
\left(-1 / 2+\vartheta_{1}, \mp 1 / 2,-1 / 2+\vartheta_{3}, \pm 1 / 2\right) & (\text { Mass })^{2}=\frac{Z_{2}}{4 \pi^{2} \alpha^{\prime}}
\end{array}
$$

This Higgs system may be understood as massive $\mathcal{N}=2$ Hypermultiplets containing respectively the $h_{i}$ and/or $H_{i}$ scalars along with the above fermions. The above scalar 
spectrum corresponds to the following mass terms in the effective potential:

$$
\begin{aligned}
V_{2}= & m_{H}^{2}\left(\left|H_{1}\right|^{2}+\left|H_{2}\right|^{2}\right)+m_{h}^{2}\left(\left|h_{1}\right|^{2}+\left|h_{2}\right|^{2}\right)+ \\
& +m_{3 H}^{2} H_{1} H_{2}+\text { h.c. }+m_{3 h}^{2} h_{1} h_{2}+\text { h.c. }
\end{aligned}
$$

where:

$$
\begin{array}{rlrl}
m_{h}{ }^{2} & =\frac{Z_{2}^{(b c)}}{4 \pi^{2} \alpha^{\prime}} ; & m_{H}{ }^{2} & =\frac{Z_{2}^{\left(b c^{*}\right)}}{4 \pi^{2} \alpha^{\prime}} \\
m_{3 h}^{2} & =\frac{1}{2 \alpha^{\prime}}\left|\vartheta_{1}^{(b c)}-\vartheta_{3}^{(b c)}\right| ; & m_{3 H}^{2}=\frac{1}{2 \alpha^{\prime}}\left|\vartheta_{1}^{\left(b c^{*}\right)}-\vartheta_{3}^{\left(b c^{*}\right)}\right|
\end{array}
$$

Notice that each of the two Higgs systems have a quadratic potential similar to that of the MSSM. In fact one also expects the quartic potential to be identical to that of the MSSM. In our case the mass parameters of the potential have an interesting geometrical interpretation in terms of the brane distances and intersection angles. In the main text we consider for simplicity the presence of just one set $h$ of Higgs multiplets. This may be achieved by appropriate choice of the wrapping numbers.

\section{B RGE solutions}

Here we display the definition of the functions appearing in the solution of the RGE in ref. [81]. We define

$$
E(t)=\left(1+\beta_{3} t\right)^{16 /\left(3 b_{3}\right)}\left(1+\beta_{2} t\right)^{3 /\left(b_{2}\right)}\left(1+\beta_{1} t\right)^{13 /\left(9 b_{1}\right)}, \quad F(t)=\int_{0}^{t} E\left(t^{\prime}\right) d t^{\prime}
$$

with $\beta_{i}=\alpha_{i}(0) b_{i} /(4 \pi)$ and $t=2 \log \left(M_{c} / M_{S S}\right)$. Here we have $\left(b_{1}, b_{2}, b_{3}\right)=(33 / 5,1,-3)$. We also define $Y_{t}=h_{t}^{2} /(4 \pi)^{2}$ with $Y_{0}=Y_{t}(0)$ and $\alpha_{0}=\alpha_{i}(0)=\alpha(0)$ for $i=2,3$, $\alpha_{1}(0)=(3 / 5) \alpha(0)$. Here $\alpha_{0}$ is the unified coupling at $M_{c}$. In our case the couplings do not strictly unify, only up to $5 \%$ corrections. In the numerical computations we take the average value of the three couplings at $M_{c}$, which is enough for our purposes. We then define the functions in eqs. (5.1), (5.2)

$$
\begin{aligned}
q(t)^{2} & =\frac{1}{\left(1+6 Y_{0} F(t)\right)^{1 / 2}}\left(1+\beta_{2} t\right)^{3 / b_{2}}\left(1+\beta_{1} t\right)^{1 / b_{1}} ; \quad h(t)=\frac{1}{2}(3 / D(t)-1) \\
k(t) & =\frac{3 Y_{0} F(t)}{D(t)^{2}} ; \quad f(t)=-\frac{6 Y_{0} H_{3}(t)}{D(t)^{2}} ; \quad D(t)=\left(1+6 Y_{0} F(t)\right) \\
e(t) & =\frac{3}{2}\left(\frac{\left(G_{1}(t)+Y_{0} G_{2}(t)\right)}{D(t)}+\frac{\left(H_{2}(t)+6 Y_{0} H_{4}(t)\right)^{2}}{3 D(t)^{2}}+H_{8}\right)
\end{aligned}
$$


and the functions $g, H_{2}, H_{3}, H_{4}, G_{1}, G_{2}$ and $H_{8}$ are independent of the top Yukawa coupling, only depend on the gauge coupling constants and are given by

$$
\begin{aligned}
g(t) & =\frac{3}{2} \frac{\alpha_{2}(0)}{4 \pi} f_{2}(t)+\frac{1}{2} \frac{\alpha_{1}(0)}{4 \pi} f_{1}(t) \\
H_{2}(t) & =\frac{\alpha_{0}}{4 \pi}\left(\frac{16}{3} h_{3}(t)+3 h_{2}(t)+\frac{13}{15} h_{1}(t)\right) \\
H_{3}(t) & =t E(t)-F(t) \\
H_{4}(t) & =F(t) H_{2}(t)-H_{3}(t) \\
H_{5}(t) & =\frac{\alpha_{0}}{4 \pi}\left(-\frac{16}{3} f_{3}(t)+6 f_{2}(t)-\frac{22}{15} f_{1}(t)\right) \\
H_{6}(t) & =\int_{0}^{t} H_{2}\left(t^{\prime}\right)^{2} E\left(t^{\prime}\right) d t^{\prime} \\
H_{8}(t) & =\frac{\alpha_{0}}{4 \pi}\left(-\frac{8}{3} f_{3}(t)+f_{2}(t)-\frac{1}{3} f_{1}(t)\right) \\
G_{1}(t) & =F_{2}(t)-\frac{1}{3} H_{2}(t)^{2} \\
G_{2}(t) & =6 F_{3}(t)-F_{4}(t)-4 H_{2}(t) H_{4}(t)+2 F(t) H_{2}(t)^{2}-2 H_{6}(t) \\
F_{2}(t) & =\frac{\alpha_{0}}{4 \pi}\left(\frac{8}{3} f_{3}(t)+\frac{8}{15} f_{1}(t)\right) \\
F_{3}(t) & =F(t) F_{2}(t)-\int_{0}^{t} E\left(t^{\prime}\right) F_{2}\left(t^{\prime}\right) d t^{\prime} \\
F_{4}(t) & =\int_{0}^{t} E\left(t^{\prime}\right) H_{5}\left(t^{\prime}\right) d t^{\prime}
\end{aligned}
$$

where $f_{i}(t)$ and $h_{i}(t)$ are defined by

$$
f_{i}(t)=\frac{1}{\beta_{i}}\left(1-\frac{1}{\left(1+\beta_{i} t\right)^{2}}\right) ; h_{i}(t)=\frac{t}{\left(1+\beta_{i} t\right)} .
$$

The low energy of the top mass may be obtained from the solutions of the one-loop renormalization group equations, divided into two pieces, SUSY and non-SUSY, i.e. (here $\left.Y_{t}=h_{t}^{2} /\left(16 \pi^{2}\right)\right)$

$$
Y_{t}\left(m_{t}\right)=Y_{t}\left(M_{S S}\right) \frac{E^{\prime}\left(t_{E W}\right)}{\left(1+(9 / 2) Y_{t}\left(M_{S S}\right) F^{\prime}\left(t_{E W}\right)\right)}
$$

where

$$
Y_{t}\left(M_{S S}\right)=\cos ^{2} \beta Y_{t}\left(M_{c}\right) \frac{E\left(t_{S S}\right)}{\left(1+6 \cos ^{2} \beta Y_{t}\left(M_{c}\right) F\left(t_{S S}\right)\right)}
$$

where the functions $E, F$ are as defined above, with $t_{S S}=2 \log \left(M_{c} / M_{S S}\right)$ and $t_{E W}=$ $2 \log \left(M_{S S} / M_{E W}\right)$. The functions $E^{\prime}, F^{\prime}$ are analogous to $E, F$ but replacing the $b_{i}$ and anomalous dimensions by the non-SUSY ones, i.e.

$$
E^{\prime}(t)=\left(1+\beta_{3}^{\prime} t\right)^{8 /\left(b_{3}^{N S}\right)}\left(1+\beta_{2}^{\prime} t\right)^{9 /\left(4 b_{2}^{N S}\right)}\left(1+\beta_{1}^{\prime} t\right)^{17 /\left(12 b_{1}^{N S}\right)}, \quad F^{\prime}(t)=\int_{0}^{t} E^{\prime}\left(t^{\prime}\right) d t^{\prime}
$$

with $\beta_{i}^{\prime}=\alpha_{i}\left(M_{S S}\right) b_{i}^{N S} /(4 \pi)$ and $t=t_{E W}$. We have now $b_{i}^{N S}=(41 / 6,-19 / 6,-7)$. For the anomalous dimensions we have made the change in the definition of $E(t)(13 / 9,3,16 / 3)$ 
$\rightarrow(17 / 12,9 / 4,8)$. Then $m_{t}\left(m_{t}\right)=h_{t}\left(m_{t}\right)<H>=h_{t}\left(m_{t}\right)(174.1) \mathrm{GeV}$. For this particular computation we take actually $t_{E W}=2 \log \left(M_{S S} /(173.2 \mathrm{GeV})\right)$.

In the case of Split-SUSY the same formulae in eq. (B.7) apply replacing $E^{\prime}, F^{\prime}$ by $E^{\prime \prime}, F^{\prime \prime}$ given by

$$
E^{\prime \prime}(t)=\left(1+\beta_{3}^{\prime \prime} t\right)^{8 /\left(b_{3}^{s p}\right)}\left(1+\beta_{2}^{\prime \prime} t\right)^{9 /\left(4 b_{2}^{s p}\right)}\left(1+\beta_{1}^{\prime \prime} t\right)^{17 /\left(12 b_{1}^{s p}\right)}, \quad F^{\prime \prime}(t)=\int_{0}^{t} E^{\prime \prime}\left(t^{\prime}\right) d t^{\prime}
$$

with $\beta_{i}^{\prime \prime}=\alpha_{i}\left(M_{S S}\right) b_{i}^{s p} /(4 \pi)$ and $t=t_{E W}$, where now $b_{i}^{s p}=(45 / 6,-19 / 6,-5)$.

\section{Wave functions and proton decay}

In F-theory GUT models most couplings of the 4d effective theory are obtained by dimensional reduction over the GUT 4-cycle $S$. In particular, to compute particle interactions one needs to consider the internal wavefunction of the $4 \mathrm{~d}$ fields of the theory, which typically have a non-trivial profile over $S$, and compute overlaps of these wavefunction such as (2.6) or (6.4).

The internal wavefunction profile of the $4 \mathrm{~d}$ particles is found by solving the corresponding equations of motion, which in turn arise from the $8 \mathrm{~d} 7$-brane action found in $[46,47]$. For $4 \mathrm{~d}$ massless fermions, one can express such internal equations of motion as a Dirac like equation, namely as [66]

$$
\mathbf{D}_{\mathbf{A}} \Psi=0
$$

with

$$
\mathbf{D}_{\mathbf{A}}=\left(\begin{array}{cccc}
0 & D_{x} & D_{y} & i \phi^{*} \\
-D_{x} & 0 & i \phi & D_{\bar{y}} \\
-D_{y} & -i \phi^{*} & 0 & -D_{\bar{x}} \\
-i \phi & -D_{\bar{y}} & D_{\bar{x}} & 0
\end{array}\right) \quad \Psi \equiv\left(\begin{array}{c}
-\sqrt{2} \eta \\
\psi_{\bar{x}} \\
\psi_{\bar{y}} \\
\chi_{x y}
\end{array}\right)
$$

where $D=\partial-i\langle A\rangle$ is the covariant derivative of the 7 -brane $8 \mathrm{~d}$ gauge theory. The components of $D$ that appear in (C.2) are along complex coordinates $(x, y)$ of the internal 4-cycle $S$ where our GUT 7-brane is wrapped. Hence, we have that $\left\langle A_{x, y}\right\rangle$ is non-zero because there are internal worldvolume fluxes $F=d A$ threading such 4-cycle, and it is precisely in this way how the hypercharge flux $F_{Y}$ as well as other fluxes enter the equations of motion. If the fermion arises from a matter curve $\Sigma_{\alpha}$ then $\phi=m^{2} f_{\alpha}(x, y)$, where $f_{\alpha}$ stands for a holomorphic function such that $\Sigma_{\alpha}=\left\{f_{\alpha}(x, y)=0\right\}$, and $m$ is a mass scale (the intersection slope) of the order of $m_{*}$. If the fermions are in the bulk of $S$ and so not related to any matter curve then $\phi=0$.

Each of the components of the vector $\Psi$ contains the degrees of freedom of a $4 \mathrm{~d}$ chiral fermion, which is related to the fact that these equations of motion arise from fermions in higher dimensions. Typically, $4 \mathrm{~d}$ chiral fermions have a vanishing component $\eta$ and the other three components non-vanishing, while the opposite happens for $4 \mathrm{~d}$ gauginos.

Let us for instance consider the case where fermionic zero modes in the representation 10 of $\mathrm{SU}(5)$ arise from a matter curve given by $\Sigma_{10}=\{y=0\}$. Let us also consider a 
worldvolume flux of the form ${ }^{14}$

$$
\langle F\rangle=i\left(M_{x} d x \wedge d \bar{x}+M_{y} d y \wedge d \bar{y}\right) Q_{10}+i(d x \wedge d \bar{y}+d y \wedge d \bar{x}) Q_{S}
$$

with $M_{x}<0<M_{y}$ and

$$
Q_{S}=\frac{1}{2}\left(N_{F} Q_{10}+N_{Y} Q_{Y}\right)
$$

where $Q_{10}$ is a gauge generator such that fermions at $\Sigma_{10}$ have charge +1 , and $Q_{Y}$ is the hypercharge generator. Here $M_{x}, M_{y}, N_{F}$ and $N_{Y}$ are flux densities, which in a local patch around $x=y=0$ one can approximate to be constant. One can then check that a zero mode solution for (C.1) is given by [85, 92]

$$
\Psi_{10}^{i}=\left(\begin{array}{c}
0 \\
\vec{v}
\end{array}\right) \psi_{10}^{i} \quad \psi_{10}^{i}=\gamma_{10}^{i} m_{*}^{4-i}(x-\zeta y)^{3-i} e^{\frac{M_{x}}{2}|x|^{2}} e^{\frac{M_{y}}{2}|y|^{2}} e^{\lambda y(\bar{y}+\zeta \bar{x})} e^{-q_{S} \operatorname{Re}(x \bar{y})}
$$

where $M_{x}<0<M_{y}, q_{S}=1 / 2\left(N_{F}+q_{Y} N_{Y}\right)$ is (C.4) evaluated for each MSSM particle inside the 10-plet, $\lambda$ is the lowest (negative) eigenvalue of the matrix

$$
\left(\begin{array}{ccc}
-M_{x} & q_{S} & 0 \\
q_{S} & -M_{y} & i m^{2} \\
0 & -i m^{2} & 0
\end{array}\right)
$$

$\vec{v}$ is the corresponding unit eigenvector and $\zeta=q_{S} /\left(\lambda+M_{x}\right)$. Finally, $\gamma_{10}^{i}$ is a normalization factor such that

$$
\left\|\Psi_{10}^{i}\right\|=m_{*}^{2} \int_{S}\left|\psi_{10}^{i}\right|^{2} d \mathrm{vol}_{S}=1
$$

and $i$ labels the wavefunction for the $i^{\text {th }}$ family of 10-plets.

As the flux (C.3) is quantized over each curve of $S$, taking the volume of $S$ reasonably large takes us to the regime $m^{2} \gg\left|M_{x}\right|,\left|M_{y}\right|,\left|N_{F}\right|,\left|N_{Y}\right|$, as usually assumed in F-theory models. In this limit $\lambda \rightarrow-m^{2}$ and $\zeta \rightarrow 0$, and so our wavefunction can be approximated by

$$
\psi_{10}^{i}=\gamma_{10}^{i} m_{*}^{4-i} x^{3-i} e^{-\frac{M}{2}|x|^{2}} e^{-m^{2}|y|^{2}-q_{S} \operatorname{Re}(x \bar{y})}
$$

with $M=\left|M_{x}\right|$.

A similar analysis can be made to solve the wavefunction for the $X, Y$ bosons, which are eigenfunctions of the Laplacian

$$
\Delta \Phi_{X, Y}=-\frac{5}{12} N_{Y} \Phi_{X, Y} \quad \Delta=\left\{D_{x}, D_{\bar{x}}\right\}+\left\{D_{y}, D_{\bar{y}}\right\}
$$

where now the covariant derivatives are constructed from the piece of the vector potential $A$ proportional to $Q_{Y}$, as the $X, Y$ bosons are neutral under $Q_{10}$. A solution to the above equation is given by

$$
\Phi_{X, Y}(x, y)=\gamma_{X, Y} m_{*} e^{-\frac{5}{24} N_{Y}\left(|x|^{2}+|y|^{2}\right)}
$$

where $\gamma_{X, Y}$ is again a normalization factor.

\footnotetext{
${ }^{14}$ See $[85]$ for more details on this F-theory model.
} 
Finally, let us compute the coupling $\Gamma_{1}^{i j}$ leading to the dimension 6 proton decay operator. As discussed in section 6, we need to compute an overlap integral of the form

$$
A_{1}=m_{*} \int_{S}\left(\Psi_{10}^{1}\right)^{*} \Psi_{10}^{1} \Phi_{X, Y}
$$

where for concreteness we have specified to the first family. The two wavefunctions $\Psi_{10}^{1}$ in (C.11) are of the form (C.5) but because of gauge invariance they must correspond to MSSM particles of the 10-plet that have different hypercharge. Hence we have $\left(q_{S}, \lambda, \zeta\right)=$ $\left(q_{S_{1}}, \lambda_{1}, \zeta_{1}\right)$ for one of them and $\left(q_{S}, \lambda, \zeta\right)=\left(q_{S_{2}}, \lambda_{2}, \zeta_{2}\right)$ for the other one. Instead of doing the $\delta$-function approximation as in the main text, let us compute the above integral with the more involved wavefunction (C.5). We find that

$$
\begin{aligned}
& A_{1}=\frac{5 N_{Y}}{12 \pi m_{*}^{2}} \frac{\left[M_{x}\left(M_{x}+1 \lambda_{1}\right)-\left(q_{S_{1}}-\lambda_{1} \zeta_{1}\right)^{2}\right]^{3 / 2}\left[M_{x}\left(M_{x}+2 \lambda_{2}\right)-\left(q_{S_{2}}-\lambda_{2} \zeta_{2}\right)^{2}\right]^{3 / 2}}{\left[\left(\frac{5}{24} N_{Y}-M_{x}\right)\left(\frac{5}{24} N_{Y}-M_{y}-\lambda_{1}-\lambda_{2}\right)-\left(\frac{1}{2}\left(q_{S_{1}}+q_{S_{2}}\right)-\lambda_{1} \zeta_{1}\right)\left(\frac{1}{2}\left(q_{S_{1}}+q_{S_{2}}\right)-\lambda_{2} \zeta_{2}\right)\right]^{3}} \\
& \frac{\left[\frac{5}{24} N_{Y}-M_{y}-\lambda_{2}-\lambda_{3}+\left(\frac{1}{2}\left(q_{S_{2}}+q_{S_{3}}\right)-\lambda_{2} \zeta_{2}\right) \zeta_{3}+\left(\frac{1}{2}\left(q_{S_{2}}+q_{S_{3}}\right)-\lambda_{3} \zeta_{3}\right) \zeta_{2}+\left(\frac{5}{24} N_{Y}-M_{x}\right) \zeta_{2} \zeta_{3}\right]^{2}}{\left(2 \zeta_{2}\left(q_{S_{2}}-\lambda_{2} \zeta_{2}\right)-M_{x} \zeta_{2}^{2}-M_{y}-2 \lambda_{2}\right)\left(2 \zeta_{3}\left(q_{S_{3}}-\lambda_{3} \zeta_{3}\right)-M_{x} \zeta_{3}^{2}-M_{y}-2 \lambda_{3}\right)}
\end{aligned}
$$

Taking the limit $M_{x}, M_{y}, N_{Y}, N_{b} \ll m^{2}$, the leading term of this expression is

$$
\left|A_{1}\right| \approx \frac{5 N_{Y}}{12 \pi m_{*}^{2}}\left(\frac{\left|M_{x}\right|}{\frac{5}{24} N_{Y}+\left|M_{x}\right|}\right)^{3}
$$

reproducing eq. (6.11) for $i=1$.

\section{References}

[1] CMS collaboration, Combination of SM Higgs Searches, PAS-HIG-11-032 (December 2011).

[2] ATLAS collaboration, Combination of Higgs Boson Searches with up to $4.9 \mathrm{fb}^{-1}$ of $\mathrm{pp}$ Collisions Data Taken at a center-of-mass energy of $7 \mathrm{TeV}$ with the ATLAS Experiment at the LHC, ATLAS-CONF-2011-163 (December 2011).

[3] H. Baer, V. Barger and A. Mustafayev, Implications of a $125 \mathrm{GeV}$ Higgs scalar for LHC SUSY and neutralino dark matter searches, Phys. Rev. D 85 (2012) 075010 [arXiv:1112.3017] [INSPIRE].

[4] L.J. Hall, D. Pinner and J.T. Ruderman, A Natural SUSY Higgs Near $126 \mathrm{GeV}$, JHEP 04 (2012) 131 [arXiv:1112.2703] [INSPIRE].

[5] A. Arbey, M. Battaglia, A. Djouadi, F. Mahmoudi and J. Quevillon, Implications of a 125 GeV Higgs for supersymmetric models, Phys. Lett. B 708 (2012) 162 [arXiv: 1112.3028] [INSPIRE].

[6] S. Akula, B. Altunkaynak, D. Feldman, P. Nath and G. Peim, Higgs Boson Mass Predictions in SUGRA Unification, Recent LHC-7 Results and Dark Matter, Phys. Rev. D 85 (2012) 075001 [arXiv:1112.3645] [INSPIRE].

[7] I. Gogoladze, Q. Shafi and C.S. Un, Higgs Boson Mass from t-b- $\tau$ Yukawa Unification, arXiv: 1112.2206 [INSPIRE]. 
[8] M. Carena, S. Gori, N.R. Shah and C.E. Wagner, A 125 GeV SM-like Higgs in the MSSM and the $\gamma \gamma$ rate, JHEP 03 (2012) 014 [arXiv:1112.3336] [INSPIRE].

[9] P. Draper, P. Meade, M. Reece and D. Shih, Implications of a 125 GeV Higgs for the MSSM and Low-Scale SUSY Breaking, Phys. Rev. D 85 (2012) 095007 [arXiv:1112.3068] [INSPIRE].

[10] J.L. Evans, M. Ibe, S. Shirai and T.T. Yanagida, A $125 \mathrm{GeV}$ Higgs Boson and Muon g-2 in More Generic Gauge Mediation, Phys. Rev. D 85 (2012) 095004 [arXiv:1201.2611] [INSPIRE].

[11] L. Aparicio, D. Cerdeno and L. Ibáñez, A 119-125 GeV Higgs from a string derived slice of the CMSSM, JHEP 04 (2012) 126 [arXiv:1202.0822] [INSPIRE].

[12] C.J. Hogan, Why the universe is just so, Rev. Mod. Phys. 72 (2000) 1149 [astro-ph/9909295] [INSPIRE].

[13] C.J. Hogan, Nuclear astrophysics of worlds in the string landscape, Phys. Rev. D 74 (2006) 123514 [astro-ph/0602104] [INSPIRE].

[14] L.J. Hall and Y. Nomura, Evidence for the Multiverse in the Standard Model and Beyond, Phys. Rev. D 78 (2008) 035001 [arXiv:0712.2454] [INSPIRE].

[15] T. Damour and J.F. Donoghue, Constraints on the variability of quark masses from nuclear binding, Phys. Rev. D 78 (2008) 014014 [arXiv:0712.2968] [INSPIRE].

[16] J.F. Donoghue, The Fine-tuning problems of particle physics and anthropic mechanisms, arXiv:0710.4080 [INSPIRE].

[17] J.F. Donoghue, K. Dutta, A. Ross and M. Tegmark, Likely values of the Higgs vacuum expectation value, Phys. Rev. D 81 (2010) 073003 [arXiv:0903.1024] [INSPIRE].

[18] N. Arkani-Hamed and S. Dimopoulos, Supersymmetric unification without low energy supersymmetry and signatures for fine-tuning at the LHC, JHEP 06 (2005) 073 [hep-th/0405159] [INSPIRE].

[19] G. Giudice and A. Romanino, Split supersymmetry, Nucl. Phys. B 699 (2004) 65 [Erratum ibid. B 706 (2005) 65] [hep-ph/0406088] [INSPIRE].

[20] L.J. Hall and Y. Nomura, A Finely-Predicted Higgs Boson Mass from A Finely-Tuned Weak Scale, JHEP 03 (2010) 076 [arXiv:0910.2235] [INSPIRE].

[21] G. Giudice and R. Rattazzi, Living Dangerously with Low-Energy Supersymmetry, Nucl. Phys. B 757 (2006) 19 [hep-ph/0606105] [INSPIRE].

[22] L.J. Hall and Y. Nomura, Spread Supersymmetry, JHEP 01 (2012) 082 [arXiv:1111.4519] [INSPIRE].

[23] C. Liu, A supersymmetry model of leptons, Phys. Lett. B 609 (2005) 111 [hep-ph/0501129] [INSPIRE].

[24] C. Liu and Z.-h. Zhao, $\theta_{13}$ and the Higgs mass from high scale supersymmetry, arXiv: 1205.3849 [INSPIRE].

[25] J. Unwin, A sharp $141 \mathrm{GeV}$ Higgs prediction from environmental selection, arXiv: 1110.0470 [INSPIRE].

[26] M. Cabrera, J. Casas and A. Delgado, Upper Bounds on Superpartner Masses from Upper Bounds on the Higgs Boson Mass, Phys. Rev. Lett. 108 (2012) 021802 [arXiv:1108.3867] [INSPIRE]. 
[27] G.F. Giudice and A. Strumia, Probing High-Scale and Split Supersymmetry with Higgs Mass Measurements, Nucl. Phys. B 858 (2012) 63 [arXiv:1108.6077] [INSPIRE].

[28] A. Arbey, M. Battaglia, A. Djouadi, F. Mahmoudi and J. Quevillon, Implications of a $125 \mathrm{GeV}$ Higgs for supersymmetric models, Phys. Lett. B 708 (2012) 162 [arXiv:1112.3028] [INSPIRE].

[29] J. Elias-Miro, J.R. Espinosa, G.F. Giudice, G. Isidori, A. Riotto and A. Strumia, Higgs mass implications on the stability of the electroweak vacuum, Phys. Lett. B 709 (2012) 222 [arXiv:1112.3022] [INSPIRE].

[30] M. Holthausen, K.S. Lim and M. Lindner, Planck scale Boundary Conditions and the Higgs Mass, JHEP 02 (2012) 037 [arXiv:1112.2415] [INSPIRE].

[31] C. Wetterich, Where to look for solving the gauge hierarchy problem?, arXiv:1112.2910 [INSPIRE].

[32] G. Degrassi, S. Di Vita, J. Elias-Miro, J.R. Espinosa, G.F. Giudice, G. Isidori and

A. Strumia, Higgs mass and vacuum stability in the Standard Model at NNLO, arXiv: 1205.6497 [INSPIRE].

[33] K. Chetyrkin and M. Zoller, Three-loop $\beta$-functions for top-Yukawa and the Higgs self-interaction in the Standard Model, JHEP 06 (2012) 033 [arXiv:1205.2892] [INSPIRE].

[34] F. Bezrukov, M.Y. Kalmykov, B.A. Kniehl and M. Shaposhnikov, Higgs boson mass and new physics, arXiv: 1205.2893 [INSPIRE].

[35] L.E. Ibáñez and A. Uranga, String Theory and Particle Physics. An Introduction to String Phenomenology, Cambridge University Press, Cambridge, U.K. (2012).

[36] M. Wijnholt, F-theory and unification, Fortsch. Phys. 58 (2010) 846 [InSPIRE].

[37] J.J. Heckman, Particle Physics Implications of F-theory, Ann. Rev. Nucl. Part. Sci. 60 (2010) 237 [arXiv:1001.0577] [InSPIRE].

[38] T. Weigand, Lectures on F-theory compactifications and model building, Class. Quant. Grav. 27 (2010) 214004 [arXiv:1009.3497] [INSPIRE].

[39] L.E. Ibáñez, From Strings to the LHC: Les Houches Lectures on String Phenomenology, arXiv: 1204.5296 [INSPIRE].

[40] R. Donagi and M. Wijnholt, Model Building with F-theory, arXiv:0802.2969 [INSPIRE].

[41] R. Blumenhagen, Gauge Coupling Unification in F-theory Grand Unified Theories, Phys. Rev. Lett. 102 (2009) 071601 [arXiv:0812.0248] [inSPIRE].

[42] J.P. Conlon and E. Palti, On Gauge Threshold Corrections for Local IIB/F-theory GUTs, Phys. Rev. D 80 (2009) 106004 [arXiv:0907.1362] [InSPIRE].

[43] C. Vafa, Evidence for F-theory, Nucl. Phys. B 469 (1996) 403 [hep-th/9602022] [INSPIRE].

[44] D.R. Morrison and C. Vafa, Compactifications of F-theory on Calabi-Yau threefolds. 1, Nucl. Phys. B 473 (1996) 74 [hep-th/9602114] [inSPIRE].

[45] D.R. Morrison and C. Vafa, Compactifications of F-theory on Calabi-Yau threefolds. 2, Nucl. Phys. B 476 (1996) 437 [hep-th/9603161] [INSPIRE].

[46] C. Beasley, J.J. Heckman and C. Vafa, GUTs and Exceptional Branes in F-theory - I, JHEP 01 (2009) 058 [arXiv:0802.3391] [INSPIRE]. 
[47] C. Beasley, J.J. Heckman and C. Vafa, GUTs and Exceptional Branes in F-theory - II: Experimental Predictions, JHEP 01 (2009) 059 [arXiv:0806.0102] [INSPIRE].

[48] R. Donagi and M. Wijnholt, Model Building with F-theory, arXiv:0802.2969 [INSPIRE].

[49] R. Donagi and M. Wijnholt, Breaking GUT Groups in F-theory, arXiv:0808.2223 [INSPIRE].

[50] M. Graña, MSSM parameters from supergravity backgrounds, Phys. Rev. D 67 (2003) 066006 [hep-th/0209200] [INSPIRE].

[51] P.G. Cámara, L. Ibáñez and A. Uranga, Flux induced SUSY breaking soft terms, Nucl. Phys. B 689 (2004) 195 [hep-th/0311241] [INSPIRE].

[52] D. Lüst, S. Reffert and S. Stieberger, Flux-induced soft supersymmetry breaking in chiral type IIB orientifolds with D3/D7-branes, Nucl. Phys. B 706 (2005) 3 [hep-th/0406092] [INSPIRE].

[53] P.G. Cámara, L. Ibáñez and A. Uranga, Flux-induced SUSY-breaking soft terms on D7-D3 brane systems, Nucl. Phys. B 708 (2005) 268 [hep-th/0408036] [INSPIRE].

[54] D. Lüst, S. Reffert and S. Stieberger, MSSM with soft SUSY breaking terms from D7-branes with fluxes, Nucl. Phys. B 727 (2005) 264 [hep-th/0410074] [INSPIRE].

[55] M. Graña, T.W. Grimm, H. Jockers and J. Louis, Soft supersymmetry breaking in Calabi-Yau orientifolds with D-branes and fluxes, Nucl. Phys. B 690 (2004) 21 [hep-th/0312232] [INSPIRE].

[56] A. Font and L. Ibáñez, SUSY-breaking soft terms in a MSSM magnetized D7-brane model, JHEP 03 (2005) 040 [hep-th/0412150] [INSPIRE].

[57] S. Dimopoulos, S. Raby and F. Wilczek, Supersymmetry and the Scale of Unification, Phys. Rev. D 24 (1981) 1681 [inSPIRE].

[58] L.E. Ibáñez and G.G. Ross, Low-Energy Predictions in Supersymmetric Grand Unified Theories, Phys. Lett. B 105 (1981) 439 [INSPIRE].

[59] S. Dimopoulos and H. Georgi, Softly Broken Supersymmetry and SU(5), Nucl. Phys. B 193 (1981) 150 [InSPIRE].

[60] Y.-J. Huo, T. Li and D.V. Nanopoulos, Canonical Gauge Coupling Unification in the Standard Model with High-Scale Supersymmetry Breaking, JHEP 09 (2011) 003 [arXiv: 1011.0964] [INSPIRE].

[61] P. Nath and P. Fileviez Perez, Proton stability in grand unified theories, in strings and in branes, Phys. Rept. 441 (2007) 191 [hep-ph/0601023] [INSPIRE].

[62] G. Aldazabal, L.E. Ibáñez, F. Quevedo and A. Uranga, D-branes at singularities: A Bottom up approach to the string embedding of the standard model, JHEP 08 (2000) 002 [hep-th/0005067] [INSPIRE].

[63] L.E. Ibáñez, F. Marchesano and R. Rabadán, Getting just the standard model at intersecting branes, JHEP 11 (2001) 002 [hep-th/0105155] [INSPIRE].

[64] M. Berkooz, M.R. Douglas and R.G. Leigh, Branes intersecting at angles, Nucl. Phys. B 480 (1996) 265 [hep-th/9606139] [INSPIRE].

[65] A. Font and L. Ibáñez, Matter wave functions and Yukawa couplings in F-theory Grand Unification, JHEP 09 (2009) 036 [arXiv:0907.4895] [INSPIRE]. 
[66] L. Aparicio, A. Font, L.E. Ibáñez and F. Marchesano, Flux and Instanton Effects in Local F-theory Models and Hierarchical Fermion Masses, JHEP 08 (2011) 152 [arXiv: 1104.2609] [INSPIRE].

[67] R. Blumenhagen, M. Cvetič and T. Weigand, Spacetime instanton corrections in $4 D$ string vacua: The Seesaw mechanism for D-brane models, Nucl. Phys. B 771 (2007) 113 [hep-th/0609191] [INSPIRE].

[68] L. Ibáñez and A. Uranga, Neutrino Majorana Masses from String Theory Instanton Effects, JHEP 03 (2007) 052 [hep-th/0609213] [INSPIRE].

[69] B. Florea, S. Kachru, J. McGreevy and N. Saulina, Stringy Instantons and Quiver Gauge Theories, JHEP 05 (2007) 024 [hep-th/0610003] [INSPIRE].

[70] R. Blumenhagen, M. Cvetič, S. Kachru and T. Weigand, D-Brane Instantons in Type II Orientifolds, Ann. Rev. Nucl. Part. Sci. 59 (2009) 269 [arXiv:0902.3251] [InSPIRE].

[71] M. Cvetič and J. Halverson, TASI Lectures: Particle Physics from Perturbative and Non-perturbative Effects in D-braneworlds, arXiv:1101.2907 [INSPIRE].

[72] S.B. Giddings, S. Kachru and J. Polchinski, Hierarchies from fluxes in string compactifications, Phys. Rev. D 66 (2002) 106006 [hep-th/0105097] [INSPIRE].

[73] V. Balasubramanian, P. Berglund, J.P. Conlon and F. Quevedo, Systematics of moduli stabilisation in Calabi-Yau flux compactifications, JHEP 03 (2005) 007 [hep-th/0502058] [INSPIRE].

[74] J.P. Conlon, F. Quevedo and K. Suruliz, Large-volume flux compactifications: Moduli spectrum and D3/D7 soft supersymmetry breaking, JHEP 08 (2005) 007 [hep-th/0505076] [INSPIRE].

[75] J.P. Conlon, D. Cremades and F. Quevedo, Kähler potentials of chiral matter fields for Calabi-Yau string compactifications, JHEP 01 (2007) 022 [hep-th/0609180] [INSPIRE].

[76] L. Aparicio, D. Cerdeno and L. Ibáñez, Modulus-dominated SUSY-breaking soft terms in F-theory and their test at LHC, JHEP 07 (2008) 099 [arXiv:0805.2943] [INSPIRE].

[77] A. Brignole, L.E. Ibáñez and C. Muñoz, Orbifold induced mu term and electroweak symmetry breaking, Phys. Lett. B 387 (1996) 769 [hep-ph/9607405] [INSPIRE].

[78] A. Brignole, L.E. Ibáñez, C. Muñoz and C. Scheich, Some issues in soft SUSY breaking terms from dilaton/moduli sectors, Z. Phys. C 74 (1997) 157 [hep-ph/9508258] [INSPIRE].

[79] A. Hebecker, A.K. Knochel and T. Weigand, A Shift Symmetry in the Higgs Sector: Experimental Hints and Stringy Realizations, JHEP 06 (2012) 093 [arXiv:1204.2551] [INSPIRE].

[80] A. Brignole, L.E. Ibáñez and C. Muñoz, Soft supersymmetry breaking terms from supergravity and superstring models, hep-ph/9707209 [INSPIRE].

[81] L.E. Ibáñez, C. Lopez and C. Muñoz, The Low-Energy Supersymmetric Spectrum According to $N=1$ Supergravity Guts, Nucl. Phys. B 256 (1985) 218 [InSPIRE].

[82] S. Weinberg, Anthropic Bound on the Cosmological Constant, Phys. Rev. Lett. 59 (1987) 2607 [INSPIRE].

[83] S. Kachru, R. Kallosh, A.D. Linde and S.P. Trivedi, de Sitter vacua in string theory, Phys. Rev. D 68 (2003) 046005 [hep-th/0301240] [INSPIRE]. 
[84] Super-Kamiokande collaboration, H. Nishino et al., Search for Proton Decay via $p \rightarrow e^{+} \pi^{0}$ and $p>\mu^{+} \pi^{0}$ in a Large Water Cherenkov Detector,

Phys. Rev. Lett. 102 (2009) 141801 [arXiv:0903.0676] [INSPIRE].

[85] A. Font et al., Non-perturbative effects and Yukawa hierarchies in local F-theory Models, to appear (2012).

[86] J.J. Heckman and C. Vafa, Flavor Hierarchy From F-theory, Nucl. Phys. B 837 (2010) 137 [arXiv:0811.2417] [INSPIRE].

[87] H. Hayashi, T. Kawano, R. Tatar and T. Watari, Codimension-3 Singularities and Yukawa Couplings in F-theory, Nucl. Phys. B 823 (2009) 47 [arXiv:0901.4941] [INSPIRE].

[88] G. Leontaris and G. Ross, Yukawa couplings and fermion mass structure in F-theory GUTs, JHEP 02 (2011) 108 [arXiv: 1009.6000] [InSPIRE].

[89] S. Cecotti, M.C. Cheng, J.J. Heckman and C. Vafa, Yukawa Couplings in F-theory and Non-Commutative Geometry, arXiv:0910.0477 [INSPIRE].

[90] J.P. Conlon and E. Palti, Aspects of Flavour and Supersymmetry in F-theory GUTs, JHEP 01 (2010) 029 [arXiv:0910.2413] [inSPIRE].

[91] F. Marchesano and L. Martucci, Non-perturbative effects on seven-brane Yukawa couplings, Phys. Rev. Lett. 104 (2010) 231601 [arXiv:0910.5496] [INSPIRE].

[92] P.G. Camara, E. Dudas and E. Palti, Massive wavefunctions, proton decay and FCNCs in local F-theory GUTs, JHEP 12 (2011) 112 [arXiv:1110.2206] [INSPIRE].

[93] Particle Data Group collaboration, K. Nakamura et al., Review of particle physics, J. Phys. G 37 (2010) 075021 [inSPIRE].

[94] M. Dine, W. Fischler and M. Srednicki, A Simple Solution to the Strong CP Problem with a Harmless Axion, Phys. Lett. B 104 (1981) 199 [INSPIRE].

[95] A. Zhitnitsky, On Possible Suppression of the Axion Hadron Interactions (In Russian), Sov. J. Nucl. Phys. 31 (1980) 260 [inSPIRE].

[96] ADMX collaboration, S. Asztalos et al., A SQUID-based microwave cavity search for dark-matter axions, Phys. Rev. Lett. 104 (2010) 041301 [arXiv:0910.5914] [INSPIRE].

[97] G.F. Giudice, R. Rattazzi and A. Strumia, Unificaxion, arXiv:1204.5465 [InSPIRE].

[98] J.P. Conlon, The QCD axion and moduli stabilisation, JHEP 05 (2006) 078 [hep-th/0602233] [inSPIRE].

[99] K. Bobkov, V. Braun, P. Kumar and S. Raby, Stabilizing All Kähler Moduli in Type IIB Orientifolds, JHEP 12 (2010) 056 [arXiv: 1003.1982] [INSPIRE].

[100] M. Cicoli, M. Goodsell and A. Ringwald, The type IIB string axiverse and its low-energy phenomenology, arXiv:1206.0819 [INSPIRE].

[101] R. Kallosh and A.D. Linde, Landscape, the scale of SUSY breaking and inflation, JHEP 12 (2004) 004 [hep-th/0411011] [INSPIRE].

[102] I. Antoniadis and S. Dimopoulos, Splitting supersymmetry in string theory, Nucl. Phys. B 715 (2005) 120 [hep-th/0411032] [INSPIRE].

[103] C. Kokorelis, Standard models and split supersymmetry from intersecting brane orbifolds, hep-th/0406258 [INSPIRE]. 
[104] I. Antoniadis, K. Benakli, A. Delgado, M. Quirós and M. Tuckmantel, Split extended supersymmetry from intersecting branes, Nucl. Phys. B 744 (2006) 156 [hep-th/0601003] [INSPIRE].

[105] D. Cremades, L. Ibáñez and F. Marchesano, More about the standard model at intersecting branes, hep-ph/0212048 [INSPIRE].

[106] A.M. Uranga, Chiral four-dimensional string compactifications with intersecting D-branes, Class. Quant. Grav. 20 (2003) S373 [hep-th/0301032] [INSPIRE].

[107] R. Blumenhagen, M. Cvetič, P. Langacker and G. Shiu, Toward realistic intersecting D-brane models, Ann. Rev. Nucl. Part. Sci. 55 (2005) 71 [hep-th/0502005] [INSPIRE].

[108] R. Blumenhagen, B. Körs, D. Lüst and S. Stieberger, Four-dimensional String Compactifications with D-branes, Orientifolds and Fluxes, Phys. Rept. 445 (2007) 1 [hep-th/0610327] [INSPIRE].

[109] F. Marchesano, Progress in D-brane model building, Fortsch. Phys. 55 (2007) 491 [hep-th/0702094] [INSPIRE]. 